\title{
Differential Detection of Gaussian MSK in a Mobile Radio Environment
}

\author{
MARVIN K. SIMON, FELlow, IEEE, AND CHARLES C. WANG, STUDENT MEMBER, IEEE
}

\begin{abstract}
Minimum shift keying with Gaussian shaped transmit pulses is a strong candidate for a modulation technique that satisfies the stringent out-of-band radiated power requirements of the mobil radio application. Numerous studies and field experiments have been conducted by the Japanese on urban and suburban mobile radio channels with systems employing Gaussian minimum-shift keying (GMSK) transmission and differentially coherent reception. A comprehensive analytical treatment is presented of the performance of such systems emphasizing the important trade-offs among the various system design parameters such as transmit and receiver filter bandwidths and detection threshold level. It is shown that two-bit differential detection of GMSK is capable of offering far superior performance to the more conventional one-bit detection method both in the presence of an additive Gaussian noise background and Rician fading.
\end{abstract}

\section{INTRODUCTION}

$\mathbf{T}^{\mathrm{H}}$ HE TRANSMISSION of voice in present-day terrestrial VHF and UHF mobile radio telephone systems is commonly accomplished by employing a single-channel-percarrier (SCPC) analog FM modulation technique [1], [2]. The same technique was also considered in the conceptual design of a land mobile satellite system (LMSS) [3]. The desire to use these same communication links for high speed data transmission as well as to provide highly secure voice has spawned an interest in digital mobile telephony, particularly amongst the Japanese [4]-[10]. Here, the SCPC structure is maintained but a spectrally efficient digital modulation is used, thus allowing the transceiver to be implemented with large scale integrated (LSI) circuit technology. Spectral efficiency is essential to maintain an acceptable level of adjacent channel interference (typically, the out-of-band power radiated to an adjacent channel should be suppressed $60-80 \mathrm{~dB}$ below that in the desired channel).

In addition to spectral efficiency, modulation methods for digital mobile radio should have constant envelope and be easy to implement. The first of these two additional requirements follows from the need to communicate over a channel which is plagued by severe multipath fading. The second follows from the desire to maintain the mobile terminal low in cost and small in size.

To satisfy a spectral efficiency requirement for allowing digital voice transmission in a narrow channel spacing, one typically requires a combination of voice coding and a bandwidth-efficient modulation. A potential candidate for the

\footnotetext{
Manuscript received March 6, 1984; revised May 20, 1984. This work was supported under a contract with the National Aeronautics and Space Administration.

The authors are with the Jet Propulsion Laboratory, California Institute of Technology, 4800 Oak Grove Drive, Pasadena, CA 91109. Telephone (818) $354-4321$.
}

former is linear predictive coding (LPC) [11] and has been suggested for use in the mobile satellite (MSAT) mobile terminal design [12]. Alternately, the Japanese have been experimenting with adaptive predictive coding-adaptive bit allocation (APC-AB) [13] and adaptive delta modulation [14]. Our interest in this paper is in the modulation scheme.

Among the many constant envelope bandwidth-efficient modulation schemes that have recently been reported in the open literature, the class of digital FM techniques known as continuous phase frequency shift keying (CPFSK) has drawn considerable attention, from which tamed FM (TFM) [15] and Gaussian baseband filtered minimum shift keying (GMSK) [7] appear to be the two most promising candidates for satisfying the stringent out-of-band radiated power requirements of the mobile radio application. Of the two, GMSK appears to be easier to implement than TFM requiring only the addition of a Gaussian baseband filter prior to the MSK modulator for shaping the transmitted RF signal spectrum. Numerous studies and field experiments have been conducted by the Japanese on urban and suburban mobile radio channels with systems employing GMSK. Both coherent and differentially coherent reception techniques have been considered with the latter broken down into one-bit and two-bit detection types [16]. Differential detection of GMSK is also being considered for the terminal design in the mobile satellite experiment (MSAT$\mathrm{X}$ ) as an update of the differential detection of MSK originally proposed for the MSAT mobile terminal in [12].

In this paper, we present a detailed analytical investigation of one- and two-bit differential detection of GMSK focusing on the inherent differences between the two which provide the significant performance advantages of one over the other previously reported by experimental means. Optimum transmit filter and receiver bandwidths (normalized by the bit rate) are determined as functions of the remaining system design parameters and the all-important selection of the detection threshold for the two-bit detection scheme is discussed and illustrated in great detail. Finally, the sensitivity of the error probability performance results to the optimization of the above-mentioned detection threshold as well as the optimum transmit and receive filter $B T$ products are also given consideration.

\section{SYSTEM MODEL-ADDITIVE WHITE GAUSSIAN NOISE (AWGN) MODEL}

Fig. 1 illustrates a GMSK transmitter consisting of (possibly) a differential encoder, a Gaussian premodulation LPF, and an FM modulator with modulation index 0.5 . The dashed 


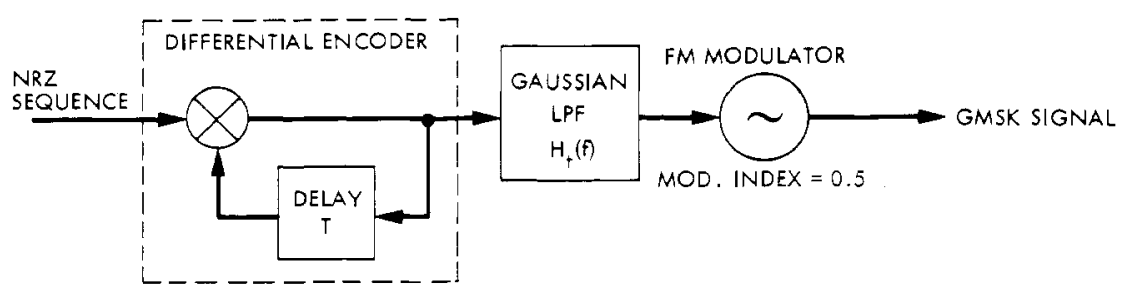

Fig. 1. A GMSK transmitter.

lines around the differential encoder indicate that it is present for a two-bit differential detector but absent for a one-bit differential detector at the receiver. As discussed in [16], this differential encoding operation is required for the two-bit detector in order that hard decisions made on the detector output reflect decisions on the true input data sequence and not a differentially decoded version of it as would be the case without the differential encoder at the transmitter input. The purpose of the Gaussian filter is to shape the input binary nonreturn to zero (NRZ) data waveform before application to the FM modulator so as to produce an RF output with constant envelope but well-suppressed out-of-band spurious power. If $B_{t}$ denotes the transmit Gaussian filter $3 \mathrm{~dB}$ bandwidth and $T$ the data symbol time, then, from a spectral standpoint, GMSK with $B_{t} T=0.2$ is almost indistinguishable from TFM [8].

Fig. 2 illustrates one- and two-bit differential detectors for GMSK (note that, structurally speaking, these are the same detectors as previously proposed for MSK [17]-[21]). The important difference between the two is as follows. The onebit detector contains a $90^{\circ}$ phase shift in the delayed arm so that the multiplier output represents the sine of the change in phase of the received signal over a one-symbol time interval, whereas the two-bit detector does not contain such a phase shift and thus the multiplier output represents the cosine of this same phase change now, however, over a two-symbol interval. As we shall soon see, and demonstrate mathematically, the impact of this difference is to result in a symmetrical eye pattern at the detector output in Fig. 2(a) and an unsymmetrical eye pattern with larger eye opening at the same point in Fig. 2(b). It is indeed this difference that suggests one to bias the detection threshold away from zero in Fig. 2(b) to take advantage of the larger eye opening thus yielding a significant improvement in error probability performance relative to the configuration in Fig. 2(a).

For either Fig. 2(a) or 2(b), the signal $x(t)$ at the input to the predetection Gaussian IF filter is

$$
x(t)=\sqrt{2 S} \cos \left[\omega_{0} t+\hat{\theta}(t)\right]+\hat{n}(t)
$$

where $S$ is the signal power, $\omega_{0}=2 \pi f_{0}$ is the center IF filter radian frequency, $\hat{n}(t)$ is the white Gaussian noise with onesided spectral density $N_{0}$, and $\hat{\theta(t)}$ is the transmit-filtered data phase after frequency modulation (with normalized deviation $h=0.5$ ) given by

$$
\hat{\theta}(t)=\frac{\pi}{2 T} \int\left[d(t)^{*} h_{t}(t)\right] d t .
$$

In (2), $d(t)$ is the effective transmitted bit stream waveform, the asterisk denotes convolution, and $h_{t}(t)$ is the impulse response of the Gaussian transmit filter, i.e.,

$$
\begin{aligned}
& h_{t}(t)=\frac{1}{\sqrt{\pi}} \alpha_{t} \exp \left[-\left(\alpha_{t} t\right)^{2}\right] \\
& \alpha_{t}=2 \sqrt{2 \pi} B_{t n}=2 \sqrt{2 \pi} \kappa B_{t} \\
& \kappa=\frac{1}{2} \sqrt{\frac{\pi}{\ln 2}}=1.0645
\end{aligned}
$$

where $k$ is the transformation coefficient relating the $3 \mathrm{~dB}$ bandwidth $B_{t}$ to the equivalent noise bandwidth $B_{t n}$. (We note that in many papers, the difference between $3 \mathrm{~dB}$ and noise bandwidth for a Gaussian filter is ignored, i.e., $\kappa$ is assumed equal to 1.0). For the one-bit differential detector of Fig. 2(a), $d(t)$ is the actual input bit stream waveform, whereas for the two-bit detector of Fig. 2(b), $d(t)$ represents a differentially encoded version of the actual input bit stream waveform.

The IF filter with equivalent low pass transfer function $H_{r}(f)$ bandlimits $x(t)$ which results in a time-varying envelope $\sqrt{2 S a}(t)$, further distorted signal phase $\phi(t)$, and a signaldependent noise term:

$$
x_{\mathrm{IF}}(t)=\sqrt{2 S} a(t) \cos \left[\omega_{0} t+\phi(t)\right]+n(t) .
$$

Letting $n_{c}(t)$ and $n_{s}(t)$ be independent low pass zero mean Gaussian random processes with variance

$$
\sigma^{2}=N_{0} \int_{-\infty}^{\infty}\left|H_{r}(f)\right|^{2} d f=N_{0} B_{r n}
$$

where $B_{r n}$ is the two-sided noise bandwidth of the equivalent low pass filter $H_{r}(f)$ (i.e., the IF bandwidth), then the noise $n(t)$ can be written in its narrow-band representation

$$
n(t)=n_{c}(t) \cos \left[\omega_{0} t+\phi(t)\right]-n_{s}(t) \sin \left[\omega_{0} t+\phi(t)\right] .
$$

Note that if the IF filter is assumed to be of the Gaussian type (as will be done later), then (3) applies with $B_{t n}$ replaced by $B_{r n} / 2$.

Substituting (6) into (4), we can write $x_{\mathrm{IF}}(t)$ in the polar form

$$
x_{\mathrm{IF}}(t)=R(t) \cos \left[\omega_{0} t+\phi(t)+\eta(t)\right]
$$

where

$$
\begin{aligned}
& R(t)=\sqrt{\left(\sqrt{2 \bar{S}} a(t)+\overline{\left.n_{c}(t)\right)^{2}+n_{s}^{2}(t)}\right.} \\
& \eta(t)=-\tan ^{-1} \frac{n_{s}(t)}{\sqrt{2 S} a(t)+n_{c}(t)} .
\end{aligned}
$$




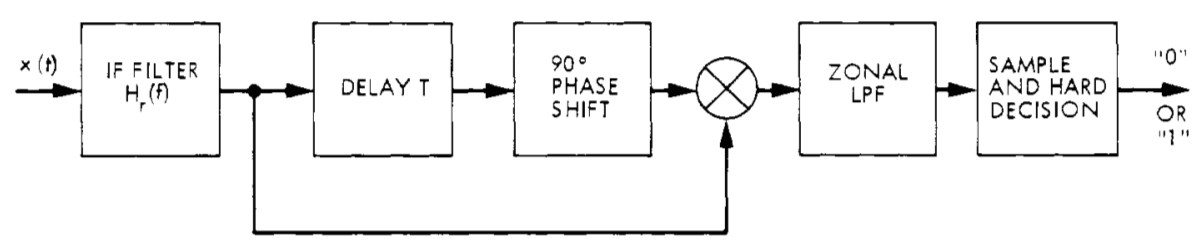

(a)

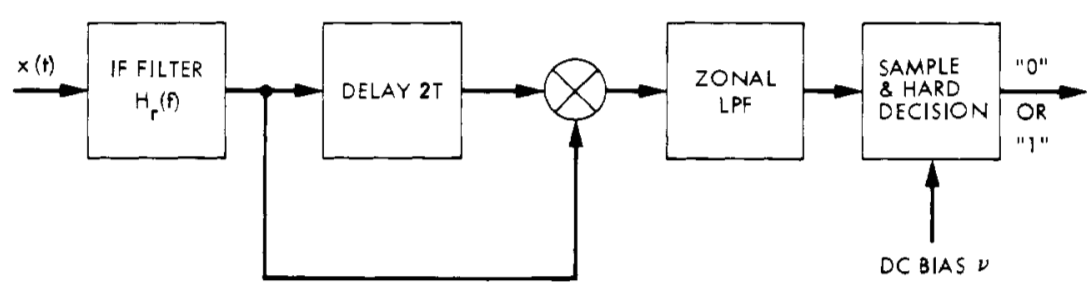

(b)

Fig. 2. (a) A one-bit differential detector for GMSK. (b) A two-bit differential detector for GMSK.

In the one-bit differential detector of Fig. 2(a), $x_{\mathrm{IF}}(t)$ of (7) is multiplied by a version of itself that is delayed by a symbol time $T$ and phase-shifted by $90^{\circ}$ resulting in (ignoring second harmonic terms)

$$
y(t)=\frac{R(t) R(t-T)}{2} \sin \left[\omega_{0} T+\Delta \Phi(T)\right]
$$

where the phase difference $\Delta \Phi(T)$ is defined by

$$
\Delta \Phi(T) \triangleq \phi(t)-\phi(t-T)+\eta(t)-\eta(t-T)
$$

and represents the change over a symbol time of the distorted signal phase plus the change in phase noise contributed by the additive white Gaussian noise. For the two-bit differential detector of Fig. 2(b), the comparable expressions to (9) and (10) are

$$
y(t)=\frac{R(t) R(t-2 T)}{2} \cos \left[2 \omega_{0} T+\Delta \Phi(2 T)\right]
$$

with

$$
\Delta \Phi(2 T)=\phi(t)-\phi(t-2 T)+\eta(t)-\eta(t-2 T) .
$$

As has been done in previous analyses of this type, we assume that the carrier frequency is chosen so that

$$
\omega_{0} T=2 \pi k, \quad k \text { integer }
$$

in which case (9) and (11) simplify to

$$
y(t)=\frac{R(t) R(t-T)}{2} \sin \Delta \Phi(T)
$$

and

$$
y(t)=\frac{R(t) R(t-2 T)}{2} \cos \Delta \Phi(2 T)
$$

The receiver of Fig. 2(a) then decides that a "1" was sent if $y(t)>0$ and a " 0 " otherwise. Since the envelope $R(t)$ is always positive, the equivalent decision rule is: decide that a "1" was sent if $\sin \Delta \Phi(T)>0$ and a "0" if $\sin \Delta \Phi(T)<0$.
We note that for the one-bit detector, the decision rule does not require the addition of a dc bias since, as will be seen shortly, the value of $\Delta \Phi(T)$ for any given data pattern is the negative of the value of $\Delta \Phi(T)$ for the complementary data pattern and the "sine" function is an odd function of its argument. Equivalently stated, complementary data sequences which, by definition, produce equal intersymbol interference to the data bit of interest, result in antipodal detector outputs and thus the eye pattern corresponding to $y(t)$ is symmetric about zero dc level.

On the contrary, for the two-bit detector of Fig. 2(b), complementary data sequences do not produce antipodal values of $\cos \Delta \Phi(2 T)$, and thus the detector output eye pattern will be asymmetric. However, because of this asymmetry, the eye opening for the two-bit detector is much larger than that of the one-bit detector. Thus, one can take advantage of this fact to improve detection performance by adding a dc bias $\nu$ to the decision threshold resulting in the decision rule: decide that a " 1 " was sent if $y(t)>\nu$ and a " 0 " otherwise. Examination of (15) reveals that this decision rule cannot be directly translated into an equivalent test of $\cos \Delta \Phi(2 T)$ against a fixed threshold because of the time dependence of the envelope product $R(t) R(t-2 T)$. To circumvent this problem, we shall assume that the IF filter output in Fig. 2(b) is first passed through a bandpass limiter (BPL) before being applied to the differential detector. Doing so normalizes the envelope $R(t)$ to unity for all $t$. Thus the above decision rule for the two bit detector can now be restated as: decide that a " 1 " was sent if $\cos \Delta \Phi(2 T)>2 \nu$ and a " 0 " otherwise.

It should be emphasized that the inclusion of a BPL in the front end of the two-bit detection receiver is more than merely an artiface to simplify the mathematics. BPL's are commonly used in practical receivers to protect various loop components, e.g., the multiplier, in environments where signal and noise (including intentional as well as unintentional interference) levels may vary over several orders of magnitude possibly exceeding the dynamic range of these components. We note, however, that despite the practical desirability of having a BPL in the one-bit detector of Fig. 2(a), from a mathematical standpoint it has no effect on system performance computed for fixed signal and noise levels. This follows from the fact 

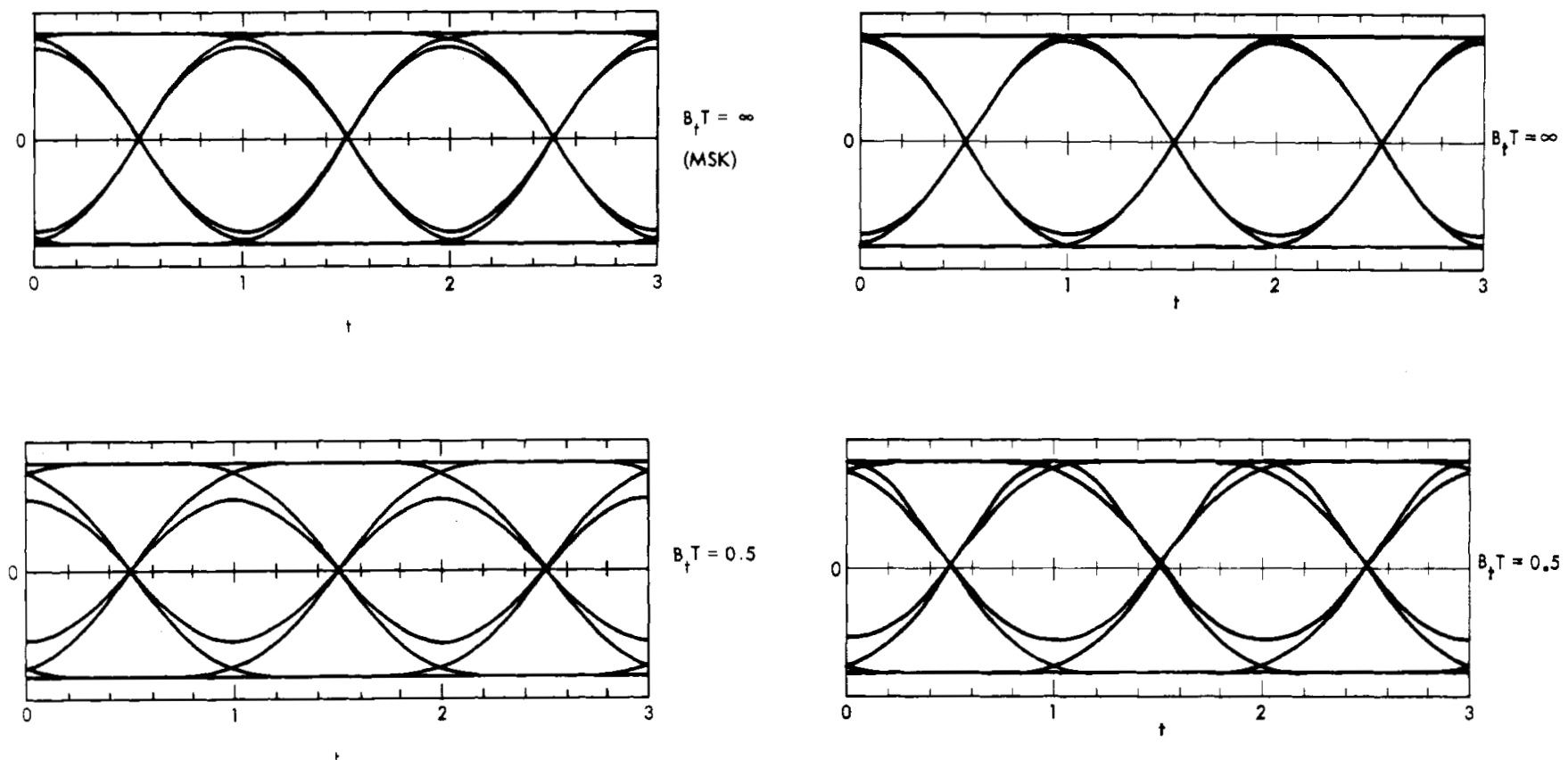

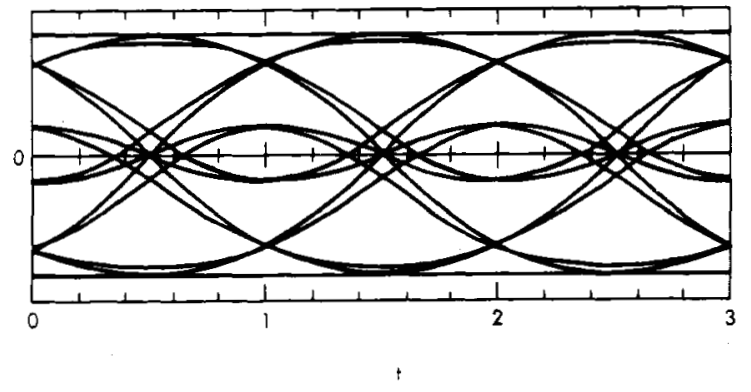

(a)
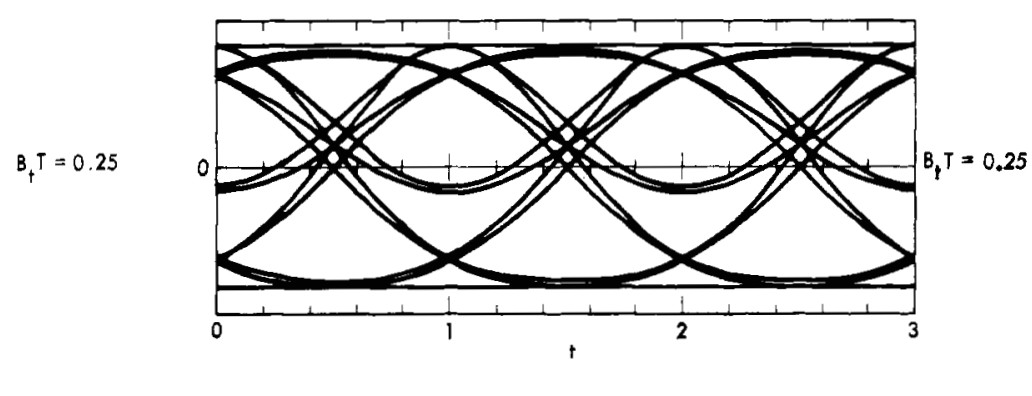

(b)

Fig. 3. (a) One-bit detector eye patterns; Gaussian IF receiver filter; $B_{r} T=1.25$. (b) Two-bit detector eye patterns; Gaussian IF receiver filter; $B_{r} T=1.25$.

that the one-bit decision rule as stated above in terms of a test of $\sin \Delta \Phi(\mathrm{T})$ against a zero threshold is independent of envelope variations in the product $R(t) R(t-T)$.

Fig. 3 is an illustration of the eye patterns for the one- and two-bit differential detector outputs (BPL's assumed in both) for a Gaussian receive filter with $B_{r} T=1.25$. This value of $B_{r} T$ is the same as that used in [16] and corresponds to the optimum value for one-bit differentially detected MSK [19]. The optimization of $B_{r} T$ for one- and two-bit differentially detected GMSK will be considered later on in this paper as well as the selection and optimization of the threshold dc bias as a function of the remaining system parameters, e.g., detection signal-to-noise and transmitter $B_{t} T$ product. Also, a more intuitive and illustrative explanation of the asymmetric behavior of the two-bit detector will be given following the mathematical characterization of the phase change $\Delta \Phi(2 T)$ in terms of the transmitted data sequence.

\section{EVALUATION OF THE AVERAGE PROBABILITY OF BIT ERROR-AWGN CHANNEL}

Based upon the above discussion, the average probability of bit error for the one-bit detector is

$$
\begin{aligned}
& \left.P_{b}=\frac{1}{2} \overline{\operatorname{Pr}\{\sin \Delta \Phi(T)<0 \mid d(t), “ 1 ”} \text { sent }\right\} \\
& +\frac{1}{2} \overline{\operatorname{Pr}\{\sin \Delta \Phi(T)>0 \mid d(t), “ 0 " \text { sent }\}}
\end{aligned}
$$

whereas for the two-bit detector we have

$$
\begin{aligned}
P_{b}= & \frac{1}{2} \overline{\operatorname{Pr}\{\cos \Delta \Phi(2 T)<2 \nu \mid d(t), ~ “ 1 " \text { sent }\}} \\
& +\frac{1}{2} \overline{\operatorname{Pr}\{\cos \Delta \Phi(2 T)>2 \nu \mid d(t), ~ " 0 " \text { sent }\} .}
\end{aligned}
$$

In the above equations, the overbar denotes statistical averaging over all equally likely input data sequences. When the transmit filter is absent (i.e., MSK modulation), it has been previously shown [21], [22] that for the range of receiver $B_{r} T$ values of interest, it is sufficient to average only over input sequences of length three symbols for the one-bit detector whereas for the two-bit detector input sequences of length four symbols are sufficient. For GMSK modulation, the additional intersymbol interference (ISI) introduced by the Gaussian 
transmit filter, in general, requires that we average over longer length input sequences depending on the value of transmitter $B_{r} T$ product. To determine these sequence lengths, we must evaluate the rectangular pulse response of the baseband transmit Gaussian filter and examine its time dispersion relative to the basic $T$-s bit interval. Using the impulse response $h_{t}(t)$ of (3), it is a simple matter to show that the response $g_{t}(t)$ of this filter to a unit amplitude rectangular pulse of duration $T \mathrm{~s}$ is given by

$$
\begin{gathered}
g_{t}(t)=Q\left(\sqrt{2} \alpha_{t} T\left(-\frac{1}{2}-\frac{t}{T}\right)\right) \\
-Q\left(\sqrt{2} \alpha_{t} T\left(\frac{1}{2}-\frac{t}{T}\right)\right) ; \\
\alpha_{t} T=5.336 B_{i} T
\end{gathered}
$$

where $Q(x)$ is the Gaussian probability integral defined by

$$
Q(x)=\frac{1}{\sqrt{2 \pi}} \int_{x}^{\infty} \exp \left(\frac{-y^{2}}{2}\right) d y .
$$

Fig. 4 illustrates the pulse responses $g_{t}(t)$ versus $t / T$ for several values of $B_{t} T$ product. One observes from these curves that the significant ISI introduced by this Gaussian pulse shaping filter extends to one adjacent (on each side) bit for $B_{t} T$ $\geq 0.4$, two adjacent bits for $0.2 \leq B_{t} T<0.4$, and four adjacent bits for $B_{t} T=0.1$. Since the shape of the transmitted GMSK pulses is rather smooth, we can assume that the additional time dispersion introduced by the receive bandpass filter is negligible as long as the receiver $B_{r} T$ product is relatively large compared to $B_{t} T$. For example, simulation results have shown that when $B_{t} T \geq 0.2$ and $B_{r} T \geq 0.75$ (the $(-\pi, \pi)$. Also in $(20),{ }^{1}$

$$
\alpha_{1}=\frac{U-r_{1} W \cos \Delta \phi}{1-r_{1}{ }^{2}} ; \quad \beta_{1}=\sqrt{\alpha_{1}^{2}-\frac{W^{2} \sin ^{2} \Delta \phi}{1-r_{1}{ }^{2}}}
$$

where $r_{1}$ is the normalized noise correlation

$$
r_{1}=\frac{\left.\mathfrak{F}^{-1}\left\{\left|H_{r}(f)\right|^{2}\right\}\right|_{t=T}}{\int_{-\infty}^{\infty}\left|H_{r}(f)\right|^{2} d f}
$$

which for a Gaussian receive IF filter

$$
H_{r}(f)=\exp \left(-\pi f^{2} / 2 B_{r n}^{2}\right) ; \quad B_{r n}=\kappa B_{r}
$$

is evaluated as

$$
r_{1}=\exp \left[-\pi\left(B_{r n} T\right)^{2}\right] .
$$

The remaining parameters $U, V$, and $W$ are defined in terms of the time-varying signal-to-noise ratio

$$
\rho(t) \triangleq \frac{a^{2}(t) S}{N_{0} B_{r n}}=\frac{E_{b}}{N_{0}} \frac{a^{2}(t)}{\kappa B_{r} T}
$$

by

$$
\begin{aligned}
& U=\frac{1}{2}[\rho(t)+\rho(t-T)] \\
& V=\frac{1}{2}[\rho(t)-\rho(t-T)]
\end{aligned}
$$

where $E_{b}=\mathrm{ST}$ is the signal bit energy and the receive filtered normalized signal amplitude as introduced in (4) is given by

$$
a(t)=\sqrt{\left(\int_{-\infty}^{\infty} h_{r}(t-\tau) \cos \hat{\theta}(\tau) d \tau\right)^{2}+\left(\int_{-\infty}^{\infty} h_{r}(t-\tau) \sin \hat{\theta}(\tau) d \tau\right)^{2}}
$$

region of most practical interest), it is sufficient to consider at most two adjacent bits on each side of the desired bit.

Returning to (16), and proceeding as in [21], the average bit error probability for the one-bit detector of Fig. 2(a) can be written in the simplified form

$$
\begin{aligned}
P_{b}= & \overline{\operatorname{Pr}\{\pi \leq \Psi(T) \leq 2 \pi \mid d(t), " \text { " } 1 \text { " sent }\}} ; \\
& \operatorname{Pr}\{\pi \leq \Psi(T) \leq 2 \pi\}=\frac{\sqrt{\alpha_{1}{ }^{2}-\beta_{1}{ }^{2}}}{2 \pi} \\
& \int_{0}^{\pi} \frac{\exp \left[-\left(\alpha_{1}-\beta_{1} \cos \theta\right]\right.}{\alpha_{1}-\beta_{1} \cos \theta} d \theta
\end{aligned}
$$

where $\Psi(T)$ is equal to $\Delta \Phi(T)$ reduced modulo $2 \pi$ which in view of the discussion following [21, eq. (14)] can be expressed as

$$
\Psi(T)=\Delta \phi(T)+\psi(T)
$$

where $\Delta \phi(T)=\phi(t)-\phi(t-T)$ and $\psi(T)=[\eta(t)-\eta(t-$ $T)$ ] $\bmod 2 \pi$ is a zero-mean random variable distributed on where $\hat{\theta}(t)$ is the filtered transmitted phase as defined in (2).

For the case of MSK, i.e., no transmit filtering, closed form expressions for $\Delta \phi, U, V$, and $W$ are easily obtainable [21] for each of the four transmitted three-bit sequences with a " 1 " as the middle bit. In particular, for the Gaussian IF filter, we have the following.

\section{A. "111" Bit Pattern}

$$
\Delta \phi=\frac{\pi}{2}, \quad U=R_{d}, \quad V=0, \quad W=\sqrt{U^{2}-V^{2}}=R_{d}
$$

\section{B. "010" Bit Pattern}

$$
\begin{aligned}
& \Delta \phi=2 \xi ; \quad \xi=\tan ^{-1} \frac{m}{1-n}<\frac{\pi}{4} \\
& U=R_{a}, \quad V=0, \quad W=R_{a} .
\end{aligned}
$$

\footnotetext{
${ }^{\prime}$ Herein we omit the dependence of $\Delta \phi$ on $T$ assuming it to be understood.
} 


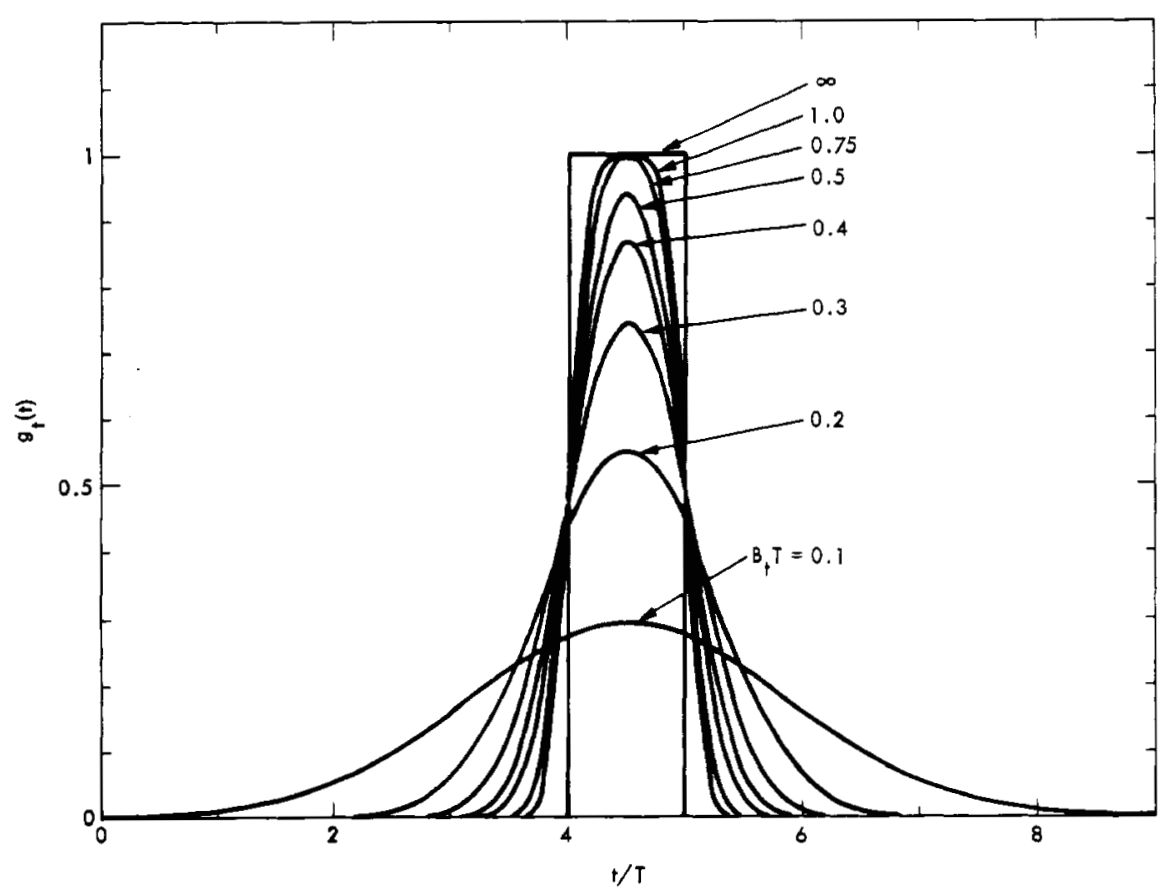

Fig. 4. Pulse response of a Gaussian filter.

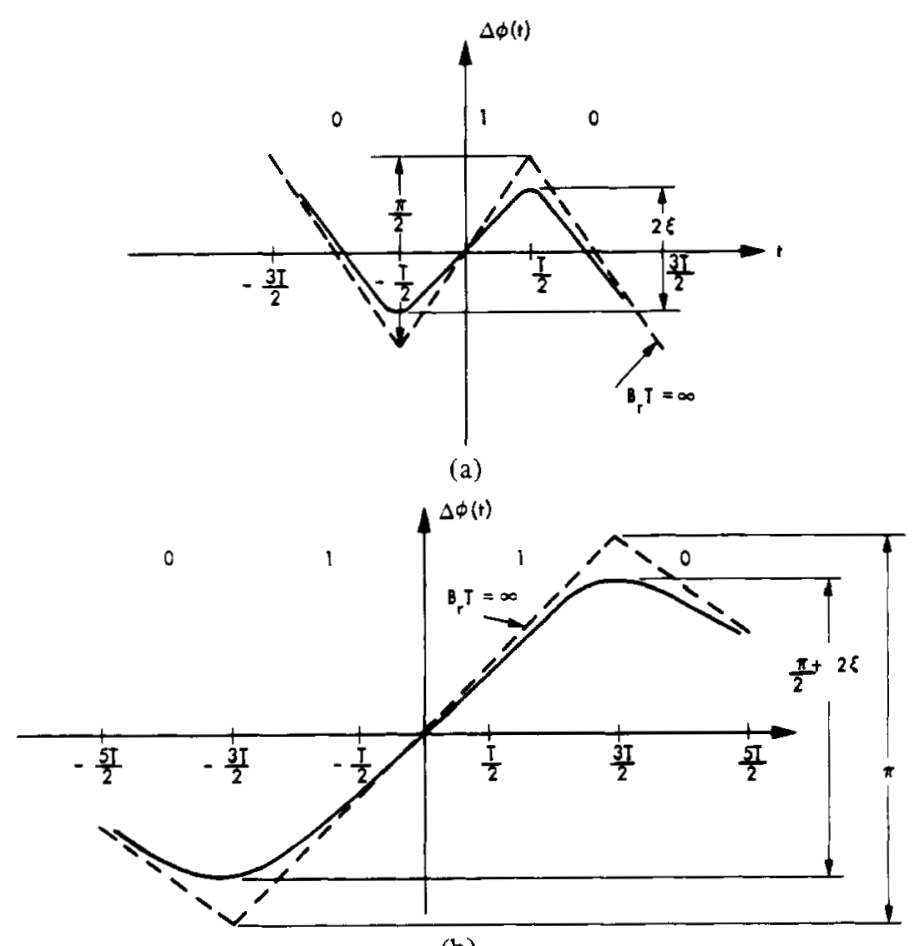

(b)

Fig. 5. (a) Worst case differential phase pattern for one-bit detection. (b) Worst case differential phase pattern for two-bit detection.

C. "011" or "110" Bit Pattern

$$
\Delta \phi=\frac{\pi}{4}+\xi
$$

$$
U=\left(R_{a}+R_{d}\right) / 2 ; \quad V=\left(R_{a}-R_{d}\right) / 2 ; \quad W=\sqrt{R_{a} R_{d}}
$$
where

$$
\begin{aligned}
& m=\frac{2}{3} \exp \left(-\frac{\pi}{8\left(B_{r n} T\right)^{2}}\right) ; n=\frac{2}{15} \exp \left(-\frac{\pi}{2\left(B_{r n} T\right)^{2}}\right) \\
& R_{a}=\frac{E_{b}}{N_{0}}\left(\frac{8}{\pi^{2}}\right) \frac{m^{2}+(1-n)^{2}}{B_{r n} T} \\
& R_{d}=\frac{E_{b}}{N_{0}} \frac{\exp \left(-\frac{\pi}{16\left(B_{r n} T\right)^{2}}\right)}{B_{r n} T}
\end{aligned}
$$

We note that the values of $\Delta \phi$ for the complementary bit patterns (i.e., those whose middle bit is a " 0 ") would, respectively, be the negatives of those in (29). Thus, a symmetrical eye pattern is obtained and the sequence causing the largest degradation of the eye is " 010 " since this sequence yields the smallest value of $\Delta \phi$ or equivalently $\sin \Delta \phi$ (see Fig. 5(a) for a typical illustration).

For GMSK, the above quantities are obtained numerically by computer evaluation for each of the 16 five-bit sequences (recall that the ISI introduced at the transmitter extends over two adjacent bits on either side of the desired bit) containing a " 1 " as the middle bit.

For the two-bit differential detector of Fig. 2(b), we can follow a procedure analogous to that presented in [22] in which case the average probability of error of (17) can be expressed as

$$
\begin{aligned}
& P_{b}=\frac{1}{2}\left[1-\overline{\operatorname{Pr}\left\{\frac{\pi}{2}-\mu \leq \Psi(2 T) \leq \frac{3 \pi}{2}+\mu \mid d(t), \text { " } 0 \text { " sent }\right\}}\right. \\
& +\overline{\left.\operatorname{Pr}\left\{\frac{\pi}{2}-\mu \leq \Psi(2 T) \leq \frac{3 \pi}{2}+\mu \mid d(t), \quad \text { “1” } \text { sent }\right\}\right]}
\end{aligned}
$$


where $\mu$ is an angle related to the detection threshold $\nu$ by

$$
\mu=\sin ^{-1} 2 \nu
$$

and analogous to (21)

$$
\Psi(2 T)=\Delta \phi(2 T)+\psi(2 T)
$$

with $\Delta \phi(2 T)=\phi(t)-\phi(t-2 T)$ and $\psi(2 T)=[\eta(t)-\eta(t$ $-2 T)] \bmod 2 \pi$. The probability required in (29) can be evaluated using the general results presented in [23] for the probability distribution of the phase angle between two vectors perturbed by Gaussian noise. In particular, applying [23, eqs. (13) and (14)], we obtain after some simplification with

$$
\begin{aligned}
\alpha_{2}{ }^{\prime}= & \frac{U-W r_{2} \sin (\mu+\Delta \phi) \sin \mu}{1-r_{2}{ }^{2} \sin ^{2} \mu} \\
\beta_{2}^{\prime}= & \operatorname{sgn}\left[W \sin (\mu+\Delta \phi)-U r_{2} \sin \mu\right] \\
& \cdot \sqrt{\alpha_{2}{ }^{2}-\frac{W^{2} \cos ^{2}(\mu+\Delta \phi)}{1-r_{2}^{2} \sin ^{2} \mu}} \\
\gamma^{\prime}= & \cos ^{-1}\left(-r_{2} \sin \mu\right)=\gamma \\
\lambda^{\prime}= & \sin ^{-1}\left(\frac{V}{\beta_{2} \sqrt{1-r_{2}^{2} \sin ^{2} \mu}}\right) .
\end{aligned}
$$

$$
\begin{aligned}
P_{b}= & \frac{1}{2} \overline{\left\{\left[F\left(\frac{\pi}{2}-\mu\right)-F\left(\frac{3 \pi}{2}+\mu\right)\right] \mid d(t), \text { “0” sent }\right\}} \\
& +\frac{1}{2}\left\{\left[F\left(\frac{3 \pi}{2}+\mu\right)-F\left(\frac{\pi}{2}-\mu\right)\right] \mid d(t), \text { " } 1 \text { " sent }\right\}
\end{aligned}
$$

where

$$
\begin{aligned}
F\left(\frac{3 \pi}{2}+\mu\right)= & \frac{W \cos (\mu-\Delta \phi)}{2 \sqrt{1-r_{2}^{2} \sin ^{2} \mu}} \\
& \cdot\left[\frac{1}{2 \pi} \int_{-\gamma-\lambda}^{\gamma-\lambda} \frac{\exp \left[-\left(\alpha_{2}-\beta_{2} \cos \theta\right)\right]}{\alpha_{2}-\beta_{2} \cos \theta} d \theta\right] \\
& -\frac{\left(r_{2} \cos \mu\right) e^{-\alpha_{2}}}{2 \sqrt{1-r_{2}^{2} \sin ^{2} \mu}} \\
& \cdot\left[\frac{1}{2 \pi} \int_{-\gamma-\lambda}^{\gamma-\lambda} \exp \left[\beta_{2} \cos \theta\right] d \theta\right]
\end{aligned}
$$

with

$$
\begin{aligned}
\alpha_{2}= & \frac{U-W r_{2} \sin (\mu-\Delta \phi) \sin \mu}{1-r_{2}^{2} \sin ^{2} \mu} \\
\beta_{2}= & \operatorname{sgn}\left[W \sin (\mu-\Delta \phi)-U r_{2} \sin \mu\right] \\
& \cdot \sqrt{\alpha_{2}^{2}-\frac{W^{2} \cos ^{2}(\mu-\Delta \phi)}{1-r_{2}^{2} \sin ^{2} \mu}} \\
\gamma= & \cos ^{-1}\left(-r_{2} \sin \mu\right) \\
\lambda= & \sin ^{-1}\left(\frac{V}{\beta_{2} \sqrt{1-r_{2}^{2} \sin ^{2} \mu}}\right)
\end{aligned}
$$

and

$$
\begin{aligned}
F\left(\frac{\pi}{2}-\mu\right)= & -\frac{W \cos (\mu+\Delta \phi)}{2 \sqrt{1-r_{2}^{2} \sin ^{2} \mu}} \\
& \cdot\left[\frac{1}{2 \pi} \int_{-\gamma^{\prime}-\lambda^{\prime}}^{\gamma^{\prime}-\lambda^{\prime}} \frac{\exp \left[-\left(\alpha_{2}^{\prime}+\beta_{2}^{\prime} \cos \theta\right)\right]}{\alpha_{2}^{\prime}-\beta_{2}^{\prime} \cos \theta} d \theta\right] \\
& +\frac{\left(r_{2} \cos \mu\right) e^{-\alpha_{2}}}{2 \sqrt{1-r_{2}{ }^{2} \sin ^{2} \mu}} \\
& \cdot\left[\frac{1}{2 \pi} \int_{-\gamma^{\prime}-\lambda^{\prime}}^{\gamma^{\prime}-\lambda^{\prime}} \exp \left[\beta_{2}^{\prime} \cos \theta\right] d \theta\right] \quad \text { (35c) }
\end{aligned}
$$

Also the parameter $r_{2}$ is the normalized noise correlation over a $2 T$ time interval and is given by (23) or (25) with $T$ replaced by $2 T$.

For MSK, closed form expressions for $\Delta \phi, U, V$, and $W$ are obtainable for the 16 transmitted four-bit sequences as follows.

\begin{tabular}{lccc}
\hline Sequence & $\Delta \phi$ & $U$ & $V$ \\
\hline 0011 & 0 & $R_{d}$ & 0 \\
1010 & 0 & $R_{a}$ & 0 \\
0010 & $-\pi / 4+\xi$ & $\left(R_{a}+R_{d}\right) / 2$ & $\left(R_{a}-R_{d}\right) / 2$ \\
1011 & $\pi / 4-\xi$ & $\left(R_{a}+R_{d}\right) / 2$ & $\left(R_{d}-R_{a}\right) / 2$ \\
1100 & 0 & $R_{d}$ & 0 \\
0101 & 0 & $R_{a}$ & 0 \\
1101 & $\pi / 4-\xi$ & $\left(R_{a}+R_{d}\right) / 2$ & $\left(R_{a}-R_{d}\right) / 2$ \\
0100 & $-\pi / 4+\xi$ & $\left(R_{a}+R_{d}\right) / 2$ & $\left(R_{d}-R_{a}\right) / 2$ \\
0000 & $-\pi$ & $R_{d}$ & 0 \\
1001 & $-\pi / 2-2 \xi$ & $R_{a}$ & 0 \\
0001 & $-3 \pi / 4-\xi$ & $\left(R_{a}+R_{d}\right) / 2$ & $\left(R_{a}-R_{d}\right) / 2$ \\
1000 & $-3 \pi / 4-\xi$ & $\left(R_{a}+R_{d}\right) / 2$ & $\left(R_{d}-R_{a}\right) / 2$ \\
1111 & $\pi$ & $R_{d}$ & 0 \\
0110 & $\pi / 2+2 \xi$ & $R_{a}$ & 0 \\
1110 & $3 \pi / 4+\xi$ & $\left(R_{a}+R_{d}\right) / 2$ & $\left(R_{a}-R_{d}\right) / 2$ \\
0111 & $3 \pi / 4+\xi$ & $\left(R_{a}+R_{d}\right) / 2$ & $\left(R_{d}-R_{a}\right) / 2$ \\
& & &
\end{tabular}

We remind the reader that because of the differential encoding operation in Fig. 1, the sequences above that contain " 01 " or " 10 " as the middle two bits correspond to a " 1 " sent whereas those that contain either " 00 " or " 11 " correspond to a " 0 ", sent. Comparing the possible values of $\cos \Delta \phi$ for " 0 " and "1" sent, we observe that they are not all antipodal. In particular, for a " 1 " sent, $\cos \Delta \phi$ can take on values of 1 or $\cos (\pi / 4-\xi)$ whereas for a " 0 "' sent the set of allowable values for $\cos \Delta \phi$ is $-1, \cos (3 \pi / 4+\xi)$ (or, equivalently, $-\cos (\pi / 4-\xi)$ ), and $\cos (\pi / 2+2 \xi$ ) (or equivalently, $-\sin 2 \xi)$. Thus, since there is no sequence corresponding to a 
" 1 " sent which gives a value $\cos \Delta \phi=\sin 2 \xi$, the eye pattern is asymmetric as previously alluded to. In fact, the transmitted sequences " 1001 " and " 0110 " which yield $\cos \Delta \phi=-\sin 2 \xi$, produce the maximum closure of the eye (see Fig. 5(b)) for a typical illustration).

For GMSK with two-bit differential detection, we must average over all 64 six-bit sequences, again recalling that the ISI introduced at the transmitter extends over two adjacent bits on either side of the bit of interest (here there are two bits of interest due to the differential encoding). As for the one-bit detection case, the values of $\Delta \phi, U, V$, and $W$ are obtained by computer evaluation.

\section{NUMERICAL RESULTS-AWGN CHANNEL}

For either one-bit or two-bit differential detection of GMSK, an optimum value of receiver $B_{r} T$ product will exist for each $B_{t} T$ product associated with the transmitter Gaussian pulse shaping filter. These optimum values of $B_{r} T$ are also a function of the level of bit error probability performance. Table I provides this optimization data for bit error probabilities of $10^{-3}$ and $10^{-6}$.

For two-bit differential detection of GMSK, we have observed that the increased eye opening allows biasing of the detection threshold to achieve improved error probability performance. This threshold can be optimized for each pair of $B_{t} T$ and $B_{r} T$ values to minimize the required $E_{b} / N_{0}$ for a given error rate. Fig. 6 illustrates the equivalent optimum threshold $2 \nu=\sin \mu$ versus $E_{b} / N_{0}$ in $\mathrm{dB}$ for various values of $B_{t} T$ and $B_{r} T=0.9$ (the optimum value for most cases in Table I). We observe that for values of $E_{b} / N_{0}$ such that the corresponding $P_{b}$ is less than $10^{-10}$, the optimum threshold approaches a saturation value. Shortly, we shall exhibit the degradation in performance obtained by fixing the threshold at these saturation values and operating the system at lower values of required $E_{b} / N_{0}$.

Fig. 7 illustrates the bit error probability performance of one-bit and two-bit differentially detected GMSK with transmitted $B_{t} T$ product as a parameter. In this figure, the receiving $B_{r} T$ product has been optimized and likewise for the detection threshold (for the two-bit detection case only). The significant performance advantage of two-bit over one-bit detection for values of $B_{t} T$ of practical interest is clearly evident. A quantitative measure of this performance improvement is given in Fig. 8 where the required $E_{b} / N_{0}$ values to achieve bit error probabilities of $10^{-3}$ and $10^{-6}$, respectively, are plotted versus the reciprocal of $B_{t} T$ with $B_{r} T$ optimized in each case. Again for the two-bit detection case, the decision threshold has also been optimized. For the purpose of comparison, the required $E_{b} / N_{0}$ to achieve the above error rates for ideal coherent detection of MSK is indicated. For an amount of transmitter pulse shaping corresponding to a value of $B_{t} T=0.25$ (typical of mobile radio applications), two-bit differentially detected GMSK offers about a $7 \mathrm{~dB}$ gain over one-bit detection. If the optimum $B_{r} T$ is not used, the degradations in performance for the same values of $P_{b}$ are illustrated in Figs. 9 and 10, respectively. Both figures show that the performance is much less sensitive when $B_{r} T$ is greater than its optimum value than when it is smaller.
TABLE I

OPTIMUM $B_{r} T$ PRODUCTS FOR DIFFERENTIAL DETECTION OF GMSK

\begin{tabular}{ccccc}
\hline \hline & \multicolumn{4}{c}{ Optimum Receiver $B_{r} T$} \\
\cline { 2 - 5 } Transmitting \\
$B_{i} T$ & \multicolumn{2}{c}{ 1-bit } & \multicolumn{2}{c}{ 2-bit } \\
\cline { 2 - 5 } & $P_{b}=10^{-3}$ & $P_{b}=10^{-6}$ & $P_{b}=10^{-3}$ & $\mathrm{P}_{\mathrm{b}}=10^{-6}$ \\
\hline$\infty$ & 1.00 & 1.15 & 0.90 & 0.95 \\
1.0 & 1.05 & 1.15 & 0.90 & 0.90 \\
0.5 & 1.10 & 1.10 & 0.90 & 0.90 \\
0.4 & 1.10 & 1.15 & 0.90 & 0.90 \\
0.32 & 1.20 & 1.20 & 0.85 & 0.85 \\
0.25 & 1.40 & 1.45 & 0.90 & 0.90 \\
\hline
\end{tabular}

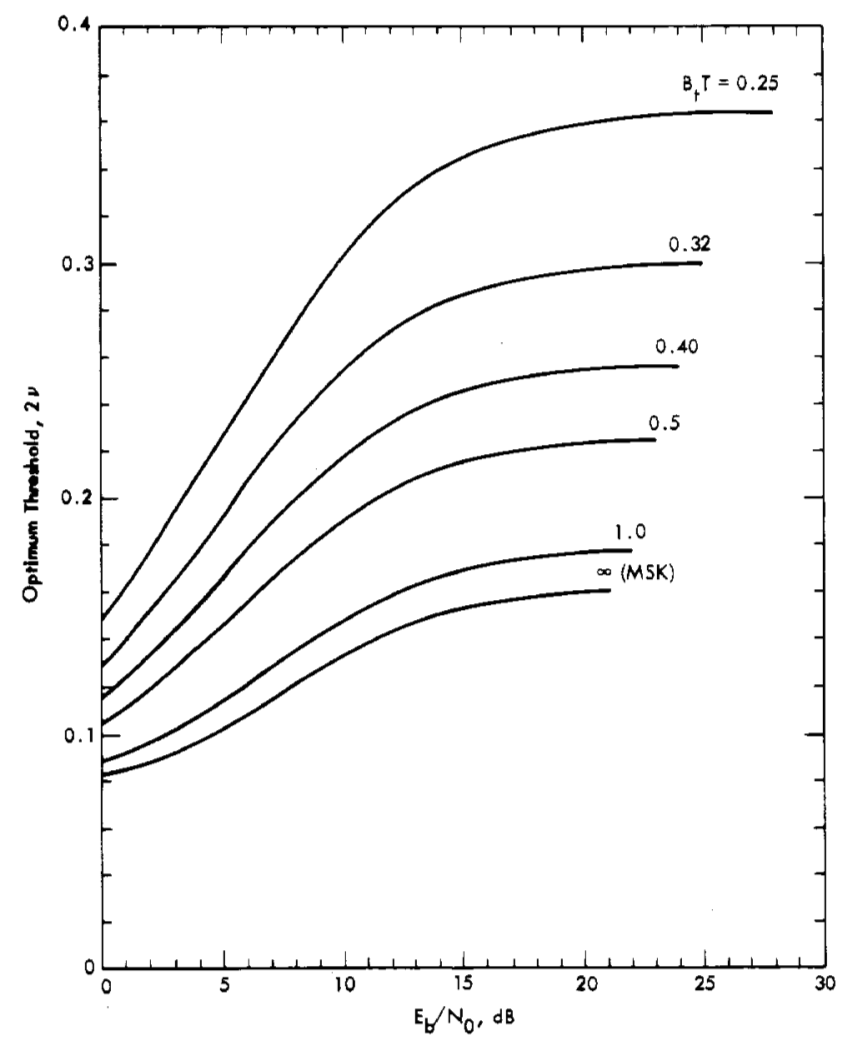

Fig. 6. Optimum threshold versus $E_{b} / N_{0}$ for two-bit differential detection of GMSK; $B_{r} \mathrm{~T}=0.9$

Figs. 11 and 12 show the performance of two-bit differentially detected GMSK for $B_{t} T=0.5$ and 0.25 , respectively, and various threshold values. In each figure, the value of $B_{r} T$ was kept fixed at 0.9 which is approximately optimum for all cases considered. For some threshold values, numbers are given in parentheses which indicate the $E_{b} / N_{0}$ values in $\mathrm{dB}$ at which the corresponding threshold is optimum. From the numerical results illustrated in these figures, we observe that by using the optimum threshold associated with large $E_{b} / N_{0}$ (i.e., the saturation value), the degradation that occurs at small $E_{b} / N_{0}$ is negligible. However, the reverse is not true, namely, using the optimum threshold associated with small $E_{b} / N_{0}$ produces significant degradation at large $E_{b} / N_{0}$ values. The implication of the above, is that for operation in a Gaussian noise environment, a practical solution for system design would be to fix the detection threshold at its large $E_{b} / N_{0}$ value 


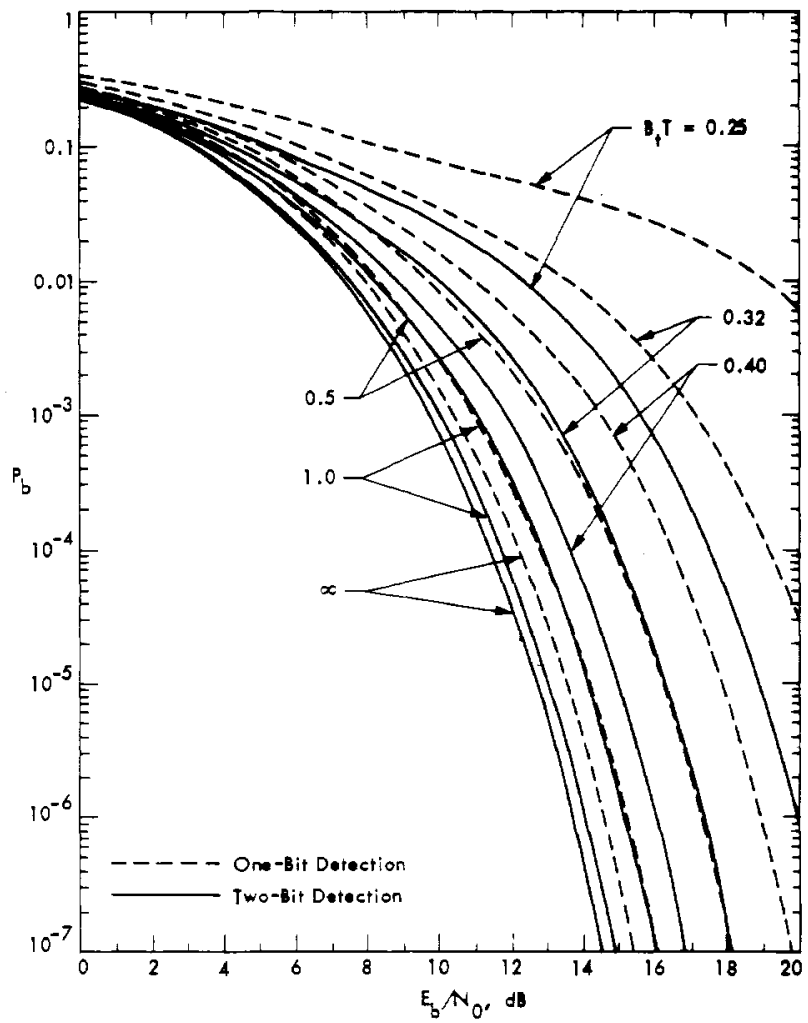

Fig. 7. Performance of one- and two-bit differential detection of GMSK; receiving $B_{r} T$ is optimized for each $B_{t} T$. (For two-bit detection case, detection threshold is optimized for each $E_{b} / N_{0}$.)

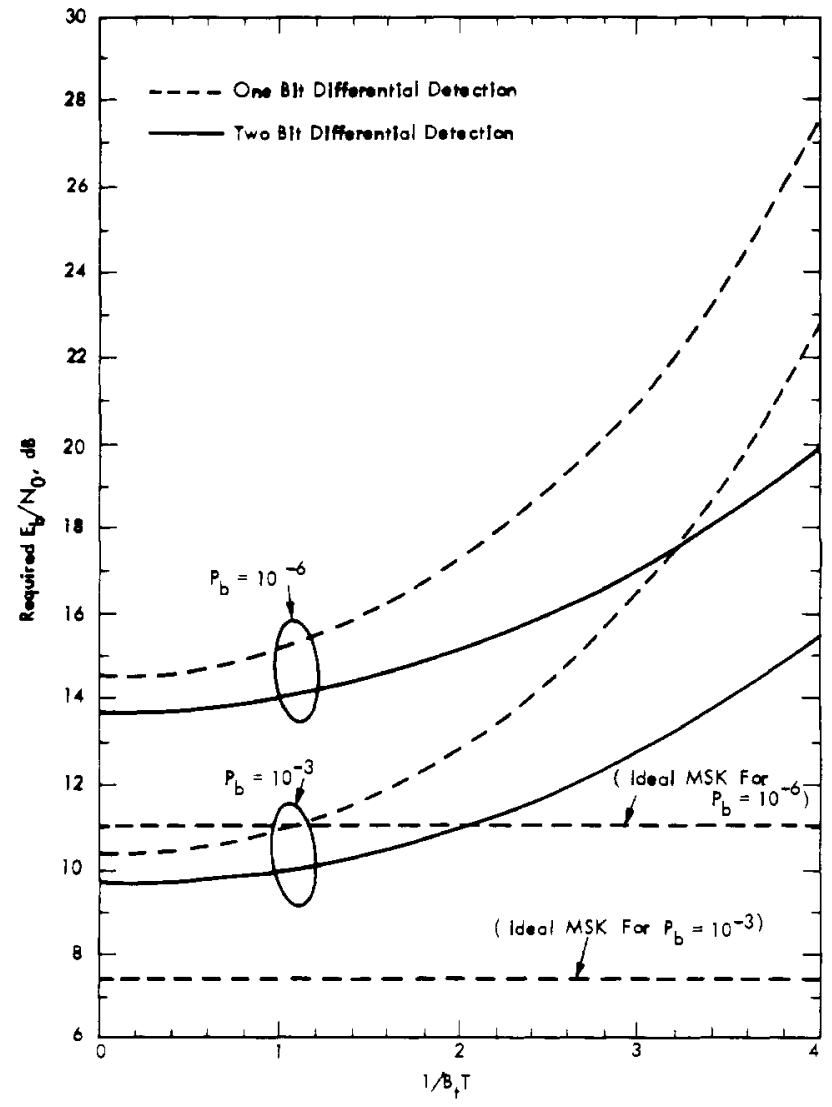

Fig. 8. Required $E_{b} / N_{0}$ in dB versus $\left(B_{t} T\right)^{-1}$ for two different values of $P_{b}$; optimum $B_{r} T$ is used along with optimum detection threshold in two-bit case.

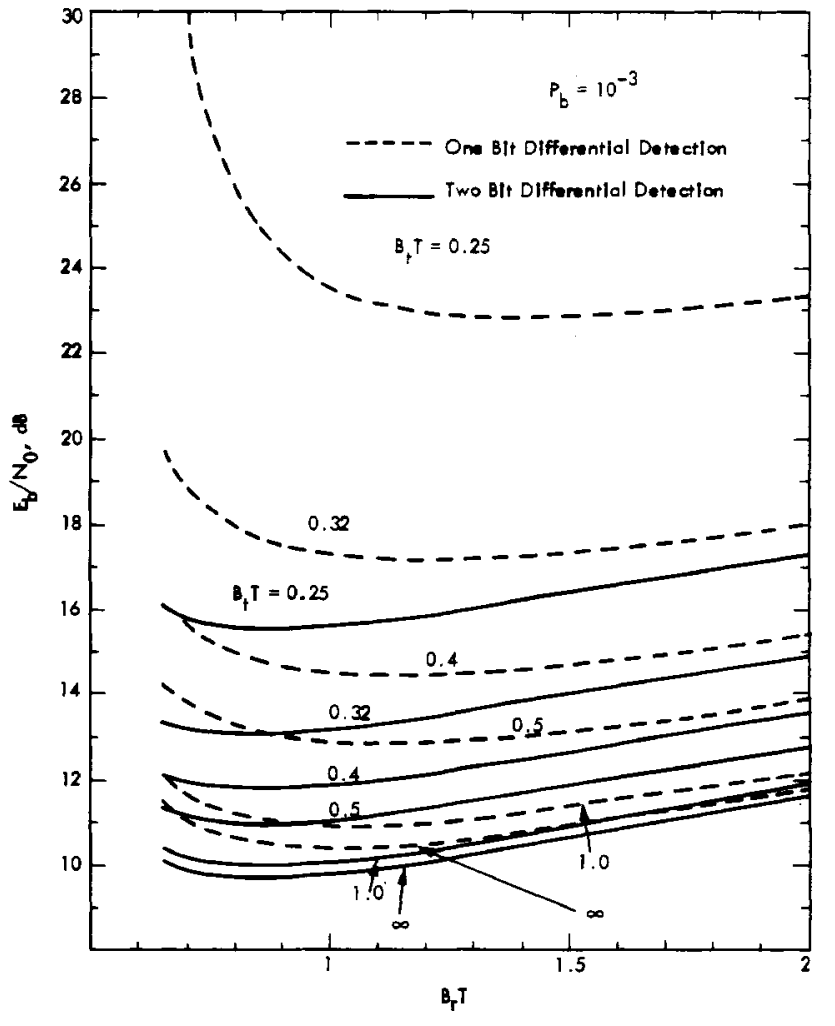

Fig. 9. Required $E_{b} / N_{0}$ in $\mathrm{dB}$ to Achieve $P_{b}=10^{-3}$ versus receiver $B_{r} T$ for different transmitter $B_{t} T$ values; optimum threshold is used for two-bit detection case.

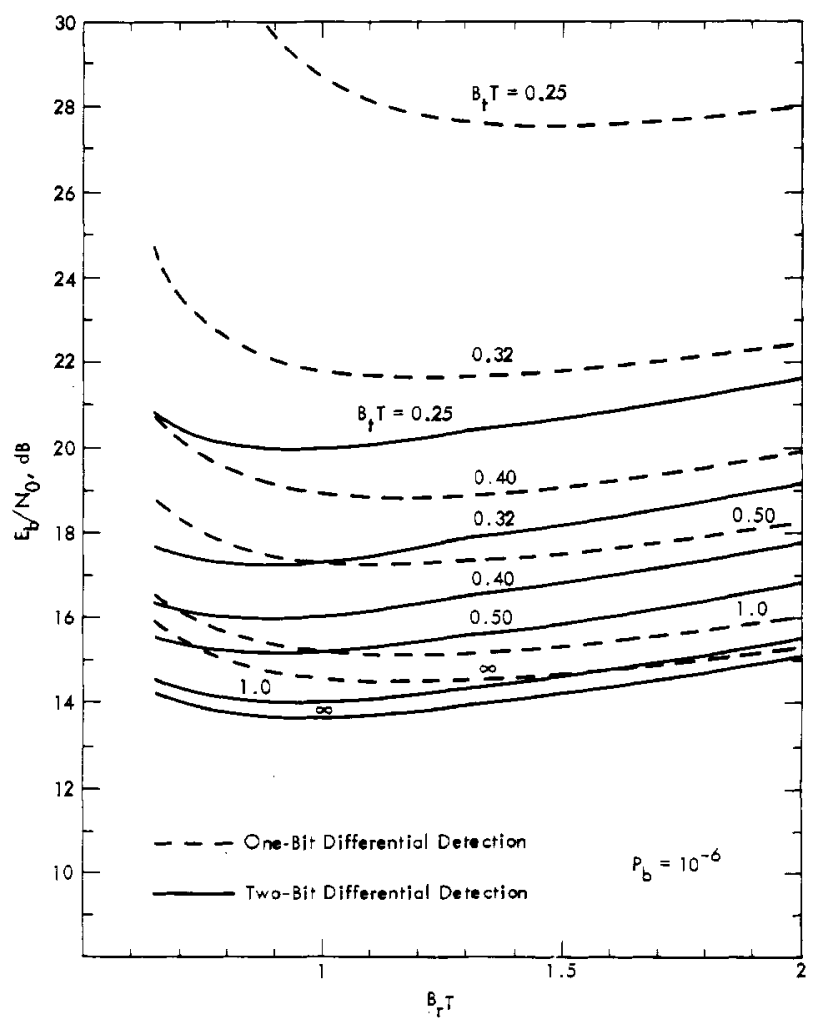

Fig. 10. Required $E_{b} / N_{0}$ in $\mathrm{dB}$ to achieve $P_{b}=10^{-6}$ versus receiver $B, T$ for different transmitter $B_{t} T$ values; optimum threshold is used for two-bit detection case. 


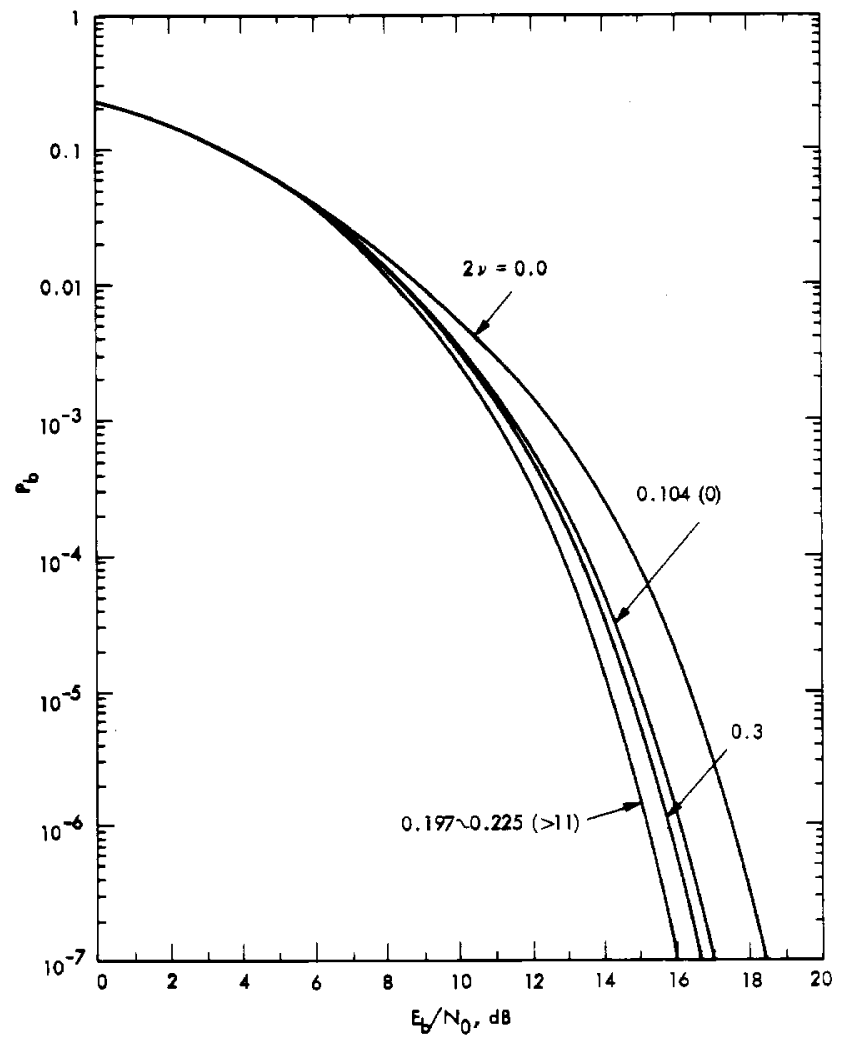

Fig. 11. Bit error probability performance of two-bit differential detection of GMSK; $B_{r} T=0.9, B_{t} T=0.5$.

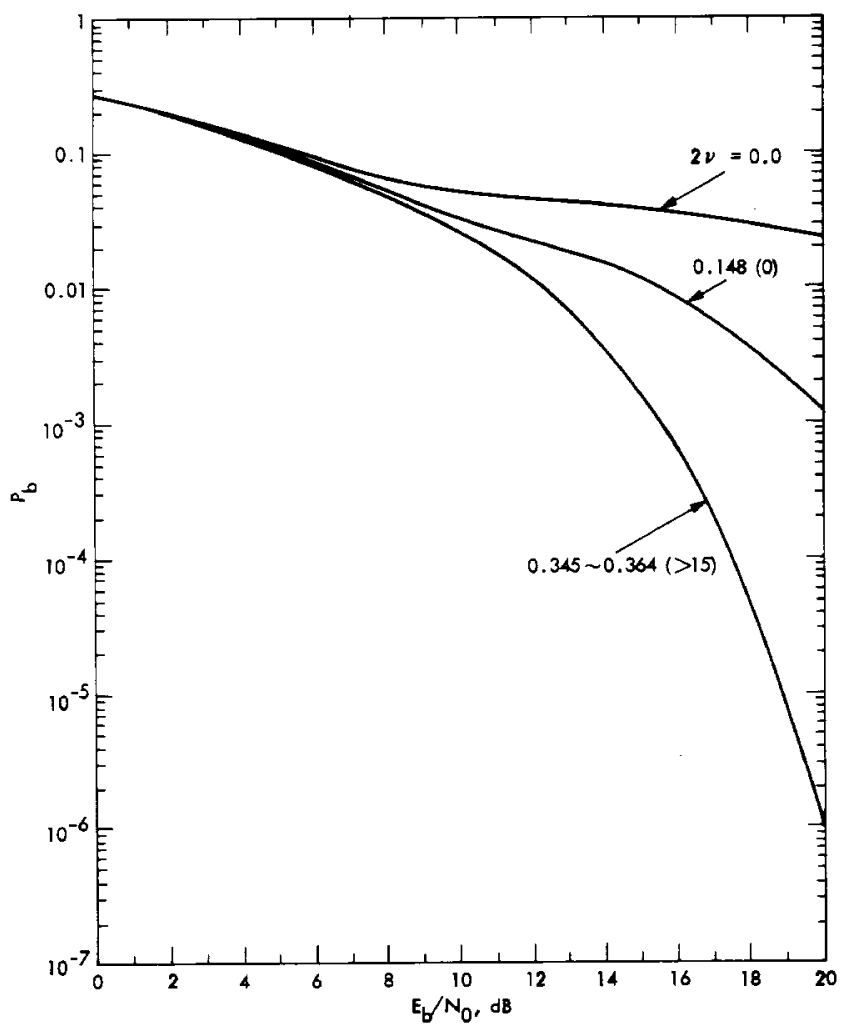

Fig. 12. Bit error probability performance of two-bit differential detection of GMSK; $B_{r} T=0.9, B_{t} T=0.25$.

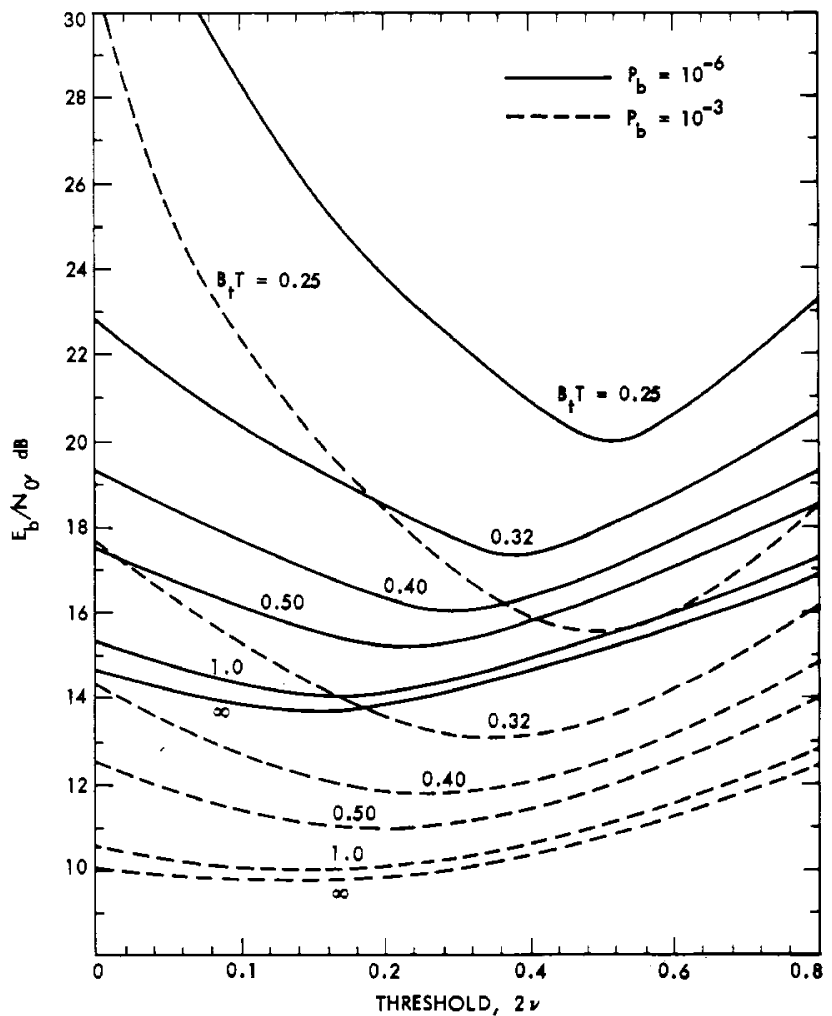

Fig. 13. Required $E_{b} / N_{0}$ in $\mathrm{dB}$ to achieve a desired bit error rate versus detection threshold; $B_{r} T=0.9$.

as determined from Fig. 6. Finally, Fig. 13 shows the sensitivity to threshold variation of the required $E_{b} / N_{0}$ necessary to achieve $P_{b}=10^{-3}$ and $10^{-6}$, respectively.

\section{PERFORMANCE OVER THE FADING CHANNEL}

In the previous sections, the authors presented the performance of one- and two-bit differential detection of GMSK in an AWGN environment. For land mobile satellite applications, the appropriate transmission model (satellite-to-mobile downlink) is that of a Rician fading channel. In this section, we extend the previous results to apply to this case.

\section{SYSTEM MODEL-RICIAN FADING CHANNEL}

When a narrow-band signal such as GMSK is transmitted through a multipath fading (time dispersive) channel, then the received signal will, in general, consist of a direct component and a summation of scattered components with random amplitudes and time delays. Assuming a flat (nonfrequency selective) fading model, then the direct component is an amplitude scaled version of the transmitted GMSK signal and the sum of the scatter components are modelled by a single diffuse component of the form of the transmitted signal with Rayleigh distributed amplitude and uniformly distributed phase. The combination of the direct and diffuse components produce an equivalent Rician channel wherein the received signal (in the absence of noise) can be written in the form

$$
s(t)=\sqrt{2} \eta \cos \left[\omega_{0} t+\theta(t)\right]
$$

where $\eta$ is a Rician random variable with probability density 
function (pdf)

$$
p(\eta)=\left\{\begin{array}{l}
\frac{2 \eta}{S_{s c}} \exp \left[-K-\frac{\eta^{2}}{S_{s c}}\right] I_{0}\left(2 \sqrt{K \eta^{2} / S_{s c}}\right), \quad \eta \geq 0 \\
0, \text { otherwise }
\end{array}\right.
$$

In (37), $S_{s c}$ is the power of the diffuse component and $K \triangleq S_{d} /$ $S_{s c}$ is the propagation signal-to-noise ratio, i.e., the ratio of the power in the direct to that in the diffuse component. Since from (36), the total average received power $\bar{S}$ is

$$
\bar{S}=\overline{\eta^{2}}=S_{d}+S_{s c}
$$

then, defining

$$
a^{2} \triangleq \frac{\eta^{2}}{S_{d}+S_{s c}}=\frac{\eta^{2}}{\bar{S}}
$$

we see from (38) that $\overline{a^{2}}=1$, and moreover, we can rewrite (36) in the normalized form

$$
s(t)=a \sqrt{2 \bar{S}} \cos \left[\omega_{0} t+\theta(t)\right]
$$

where the normalized amplitude " $a$ "' has the pdf through the details, we arrive at the result

$$
\overline{\exp \left(-C a^{2}\right)}=\frac{1+K}{1+K+C} \exp \left\{-K \frac{C}{1+K+C}\right\} \text {. }
$$

Note that as $K \rightarrow 0$ (i.e., pure Rayleigh fading), we arrive at the well-known result

$\overline{\exp \left(-C a^{2}\right)}=\frac{1}{1+C}$.

\section{EVALUATION OF THE ERROR PROBABILITY OF BIT ERROR-RICIAN FADING CHANNEL}

For one-bit differential detection of GMSK, the average bit error probability over the AWGN was shown to be given by (20) where $\alpha_{1}$ and $\beta_{1}$ are parameters (see (22)) that are linearly dependent on the bit energy-to-Gaussian noise ratio $E_{b} / N_{0}$ through the relations (23)-(30). Since, in view of this, the quantity $\sqrt{\alpha_{1}{ }^{2}-\beta_{1}^{2}} /\left(\alpha_{1}-\beta_{1} \cos \theta\right)$ is independent of $E_{b} / N_{0}$, then using (43), we obtain the analogous result for performance over the Rician channel, namely,

$$
\begin{aligned}
& P_{b}=\frac{\overline{(1+K) \sqrt{\bar{\alpha}_{1}^{2}-\bar{\beta}_{1}^{2}}}}{2 \pi} \int_{0}^{\pi} \frac{\left[1+K+\bar{\alpha}_{1}-\bar{\beta}_{1} \cos \theta\right]^{-1}}{\bar{\alpha}_{1}-\bar{\beta}_{1} \cos \theta} \\
& \cdot \exp \left(-K \frac{\bar{\alpha}_{1}-\bar{\beta}_{1} \cos \theta}{1+K+\bar{\alpha}_{1}-\bar{\beta}_{1} \cos \theta}\right) d \theta
\end{aligned}
$$

$$
p(a)=\left\{\begin{array}{l}
2 a(1+K) \exp \left[-K-a^{2}(1+K)\right] I_{0}(2 a \sqrt{K(1+K)}), a \geq 0 \\
0, \quad \text { otherwise }
\end{array}\right.
$$

In what follows, we shall need to perform expections of the form

$$
\overline{\exp \left(-C a^{2}\right)} \triangleq \int_{-\infty}^{\infty} p(a) \exp \left(-C a^{2}\right) d a
$$

where $C$ is a constant. Substituting (41) into (42) and carrying where $\bar{\alpha}_{1}$ and $\bar{\beta}_{1}$ are related to the average bit energy-to-noise ratio $\bar{E}_{b} / N_{0}=\bar{S} T / N_{0}$ by the identical set of relationships as mentioned above.

For the case of two-bit differential detection of GMSK, we can use similar analogous arguments to transform (34) and (35) into

$$
\begin{aligned}
& P_{b}=\frac{1}{2}\left\{\left[F\left(\frac{\pi}{2}-\mu\right)-F\left(\frac{3 \pi}{2}+\mu\right)\right] \mid d(t), “ 0 \text { " sent }\right\} \\
&+\frac{1}{2}\left\{\left[F\left(\frac{3 \pi}{2}+\mu\right)-F\left(\frac{\pi}{2}-\mu\right)\right] \mid d(t), \text { " } 1 \text { ", sent }\right\} \\
& F\left(\frac{3 \pi}{2}+\mu\right)= \frac{(1+K) \bar{W} \cos (\mu-\Delta \phi)}{2 \sqrt{1-r_{2}{ }^{2} \sin ^{2} \mu}} \\
& \cdot\left[\frac{1}{2 \pi} \int_{-\gamma-\lambda}^{\gamma-\lambda} \frac{\exp \left\{-K\left(\frac{\bar{\alpha}_{2}-\bar{\beta}_{2} \cos \theta}{1+K+\bar{\alpha}_{2}-\bar{\beta}_{2} \cos \theta}\right)\right\}}{\left(\bar{\alpha}_{2} \cos \theta\right)\left(1+K+\bar{\alpha}_{2}-\bar{\beta}_{2} \cos \theta\right)} d \theta\right] \\
&-\frac{(1+K)\left(r_{2} \cos \mu\right)}{2 \sqrt{1-r_{2}{ }^{2} \sin ^{2} \mu}} \\
& \cdot\left[\frac{1}{2 \pi} \int_{-\gamma-\lambda}^{\gamma-\lambda} \frac{\exp \left\{-K\left(\frac{\bar{\alpha}_{2}-\bar{\beta}_{2} \cos \theta}{1+K+\bar{\alpha}_{2}-\bar{\beta}_{2} \cos \theta}\right)\right\}}{1+K+\bar{\alpha}_{2}-\bar{\beta}_{2} \cos \theta} d \theta\right]
\end{aligned}
$$


and

$$
\begin{aligned}
& F\left(\frac{\pi}{2}-\mu\right)=-\frac{(1+K) \bar{W} \cos (\mu+\Delta \phi)}{2 \sqrt{1-r_{2}^{2} \sin ^{2} \mu}} \\
& {\left[\frac{1}{2 \pi} \int_{-\gamma^{\prime}-\lambda^{\prime}}^{\gamma^{\prime}-\lambda^{\prime}} \frac{\exp \left\{-K\left(\frac{\bar{\alpha}_{2}^{\prime}-\overline{\bar{\beta}}_{2}^{\prime} \cos \theta}{1+K+\bar{\alpha}_{2}^{\prime}-\bar{\beta}_{2}^{\prime} \cos \theta}\right)\right\}}{\left(\bar{\alpha}_{2}^{\prime}-\bar{\beta}_{2}^{\prime} \cos \theta\right)\left(1+K+\bar{\alpha}_{2}{ }^{\prime}-\bar{\beta}_{2}{ }^{\prime} \cos \theta\right)} d \theta\right]} \\
& +\frac{(1+K)\left(r_{2} \cos \mu\right)}{2 \sqrt{1-r_{2}^{2} \sin ^{2} \mu}} \\
& \cdot\left[\frac{1}{2 \pi} \int_{-\gamma^{\prime}-\lambda^{\prime}}^{\gamma^{\prime}-\lambda^{\prime}} \frac{\exp \left\{-K\left(\frac{\bar{\alpha}_{2}{ }^{\prime}-\bar{\beta}_{2}{ }^{\prime} \cos \theta}{1+K+\bar{\alpha}_{2}{ }^{\prime}-\bar{\beta}_{2}{ }^{\prime} \cos \theta}\right)\right\}}{1+K+\bar{\alpha}_{2}{ }^{\prime}-\bar{\beta}_{2}{ }^{\prime} \cos \theta} d \theta\right] .
\end{aligned}
$$

\section{NUMERICAL RESULTS-RICIAN FADING CHANNEL}

Figs. 14 and 15 illustrate the average bit error probability of one-bit differential detection of GMSK as computed from (44), (45), and (46) for transmit $B_{t} T$ products equal to 0.25 and 0.5 , respectively. The receive filter $B_{r} T$ product is kept fixed at the corresponding optimum value as determined from Table I. At each point on the two-bit detection curves, the optimum threshold (that which minimizes $P_{b}$ ) has been selected. The curves marked "no fading" represent the performance in an AWGN channel and thus agree with the equivalent numerical results given in Fig. 7. As fading increases, the performance deteriorates as expected approaching the familiar inverse linear behavior of error rate with average bit energy-to-noise ratio in the limit as the direct signal component disappears, i.e., a purely Rayleigh channel. Furthermore, the dramatic improvement in performance on the AWGN offered by two-bit over one-bit differential detection diminishes as fading begins to dominate the channel.

To demonstrate the sensitivity of two-bit differential detection performance to detection threshold, Figs. 16 and 17 illustrate the required average bit energy-to-noise ratio to achieve bit error rates of $10^{-3}$ and $10^{-6}$ for $B_{r} T=0.9$ and $B_{t} T=0.25,0.5$, respectively. We observe from these results that the selection of the optimum threshold (that which minimizes required $E_{b}$ / $N_{0}$ ) is virtually insensitive to the fading level. Furthermore, the sensitivity of performance degradation to threshold choices other than the optimum is much less for the fading channel than for the AWGN case. Thus, one may select the optimum threshold for the AWGN channel and suffer virtually no loss in optimality on the fading channel. This is a very desirable result from the practical standpoint of system design.

\section{CONCLUSION}

Two-bit differential detection of GMSK is capable of offering far superior performance to the more conventional one-bit differential detection of the same modulation. The potential advantage stems from the asymmetry of the eye pattern produced at the detector output (one-bit detection has a

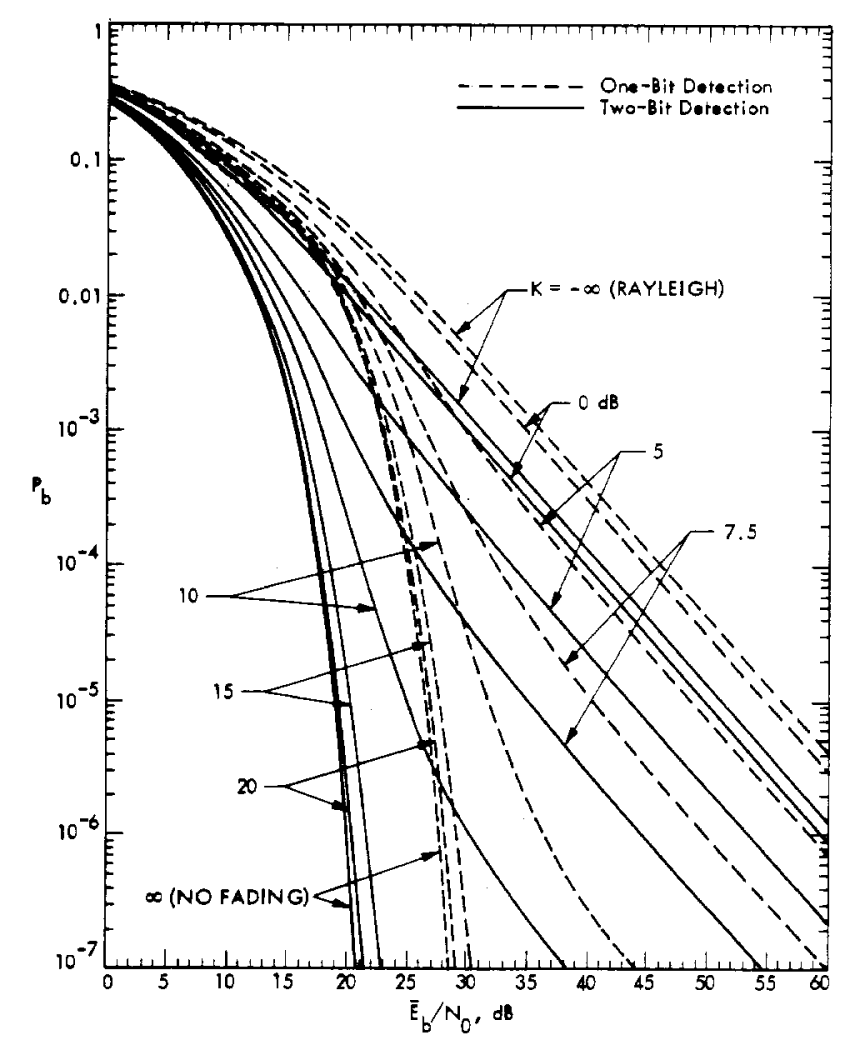

Fig. 14. Average bit error probability performance of one- and two-bit differential detection of GMSK over Rician fading channel; $B_{i} T=0.25$, $B_{r} T=1.4$ for one-bit and 0.9 for two-bit detection. (For two-bit case, detection threshold is optimized at each $\bar{E}_{b} / N_{0}$.)

symmetrical eye pattern with a smaller eye opening) thus enabling one to bias the decision threshold in the direction of the larger opening of the eye. A thorough parametric investigation of the two-bit differential detection scheme reveals that the best performance is obtained by optimizing the receiver filter bandwidth-time product as well as the detection threshold. The sensitivity of the receiver performance to deviations from these optimum parameter values has also been explored in great numerical detail. 


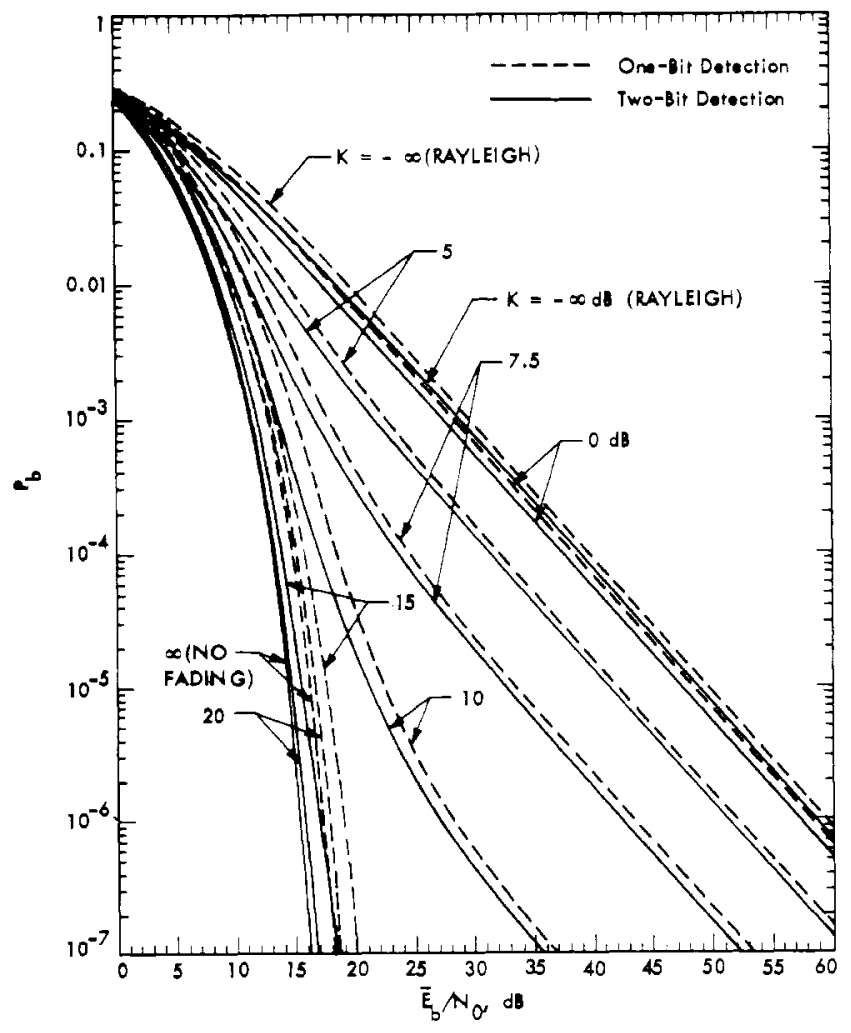

Fig. 15. Average bit error probability performance of one- and two-bit differential detection of GMSK over Rician fading channel; $B_{t} T=0.5$, $B_{r} T=1.1$ for one-bit and 0.9 for two-bit detection. (For two-bit case, detection threshold is optimized at each $\bar{E}_{b} / N_{0}$.)

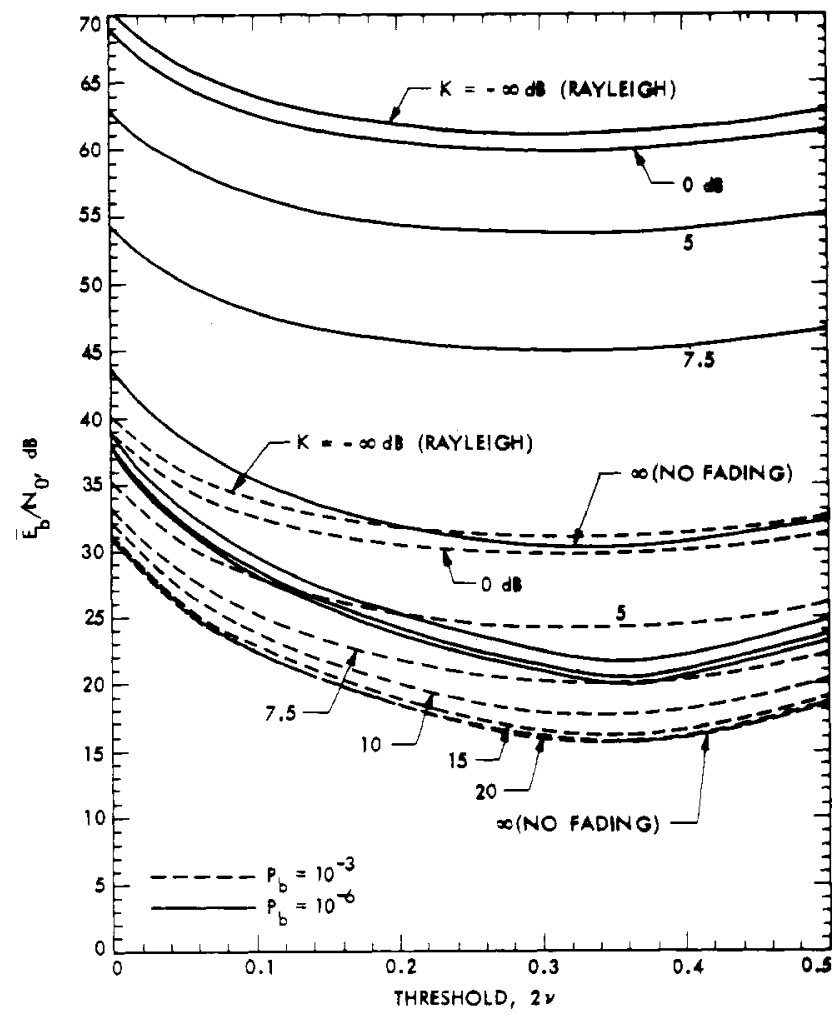

Fig. 16. Required $\bar{E}_{b} / N_{0}$ to achieve a desired error rate versus detection threshold; $B_{t} T=0.25 ; B_{r} T=0.9$.

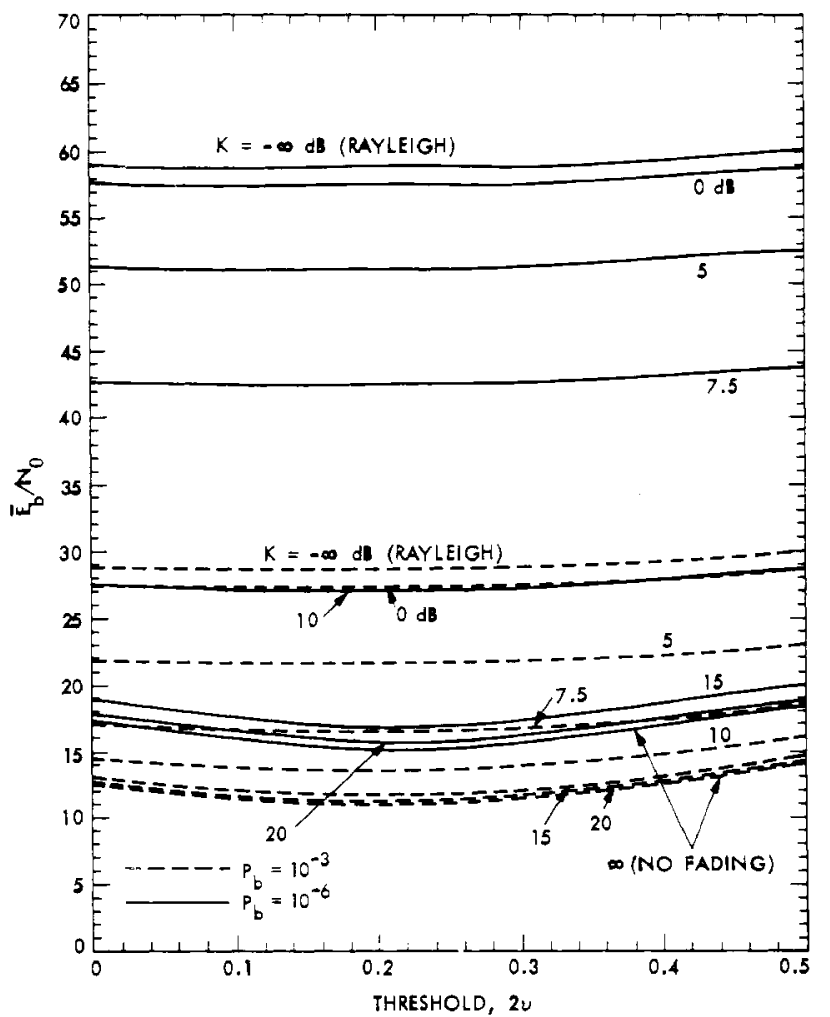

Fig. 17. Required $\bar{E}_{b} / N_{0}$ to achieve a desired error rate versus detection threshold; $B_{t} T=0.5 ; B_{r} T=0.9$.

\section{REFERENCES}

[1] S. Ito and Y. Matsuzaka, " $800 \mathrm{MHz}$ band land mobile telephone system-Overall reaction review," Rev. ECL, vol. 25 , no. 6, p. 1147 , Nov./Dec. 1977.

[2] F. H. Blecher, "Advanced mobile phone service," IEEE Trans. Veh. Technol., vol. VT-29, no. 2, pp. 238-244, May 1980.

[3] F. Naderi, Ed., "Land mobile satellite service (LMSS)," JPL Pub. 8219, Jet Propulsion Lab., Pasadena, CA, Feb. 15, 1982.

[4] H. M. Sachs, "Digital voice considerations for the land mobile radio services," in IEEE 27th Veh. Technol. Conf. Rec., Mar. 1977, pp. 207-219.

[5] K. Hirade and M. Ishizuka, "Feasabilty study of digital voice transmission in mobile radio communications," Trans. IECE Japan, vol. CS-78-2, pp. 9-16, Apr. 1978.

[6] K. Murota and K. Hirade, "Study of modulation for digital mobile telephony," in IEEE 29th Veh. Technol. Conf. Rec., Mar. 1979, pp. 13-19.

[7] - "GMSK modulation for digital mobile radio telephony," IEEE Trans. Commun., vol. COM-29, pp. 1044-1050, July 1981

[8] K. Murota, K. Kinoshita, and K. Hirade, "Spectrum efficiency of GMSK land mobile radio," in ICC'81 Conf. Rec., June 1981, pp. 23.8.1-23.8.5.

[9] K. Daikoku, K. Murota, and K. Momma, "High-speed digital transmission experiment in $920 \mathrm{MHz}$ in urban and suburban mobile radio channels," IEEE Trans. Veh. Technol., vol. VT-31, pp. 70-75, May 1982.

[10] K. Hirade, K. Murota, and M. Hata, "GMSK transmission performance in land mobile radio," GLOBECOM'82, pp. B3.4.1-B.3.4.5, Dec. 1982.

[11] Y. Makhoul, "Linear prediction: A tutorial review," Proc. IEEE, vol. 63, pp. 561-580, April 1975.

[12] J. E. Nicholson, J. D. B. Kent, and J. T. Sydor, "MSAT mobile terminal design considerations," in IEEE 33rd Veh. Technol. Conf. Rec., May 1983, pp. 347-351.

[13] M. Honda and F. Itakura, "Periodic coding of speech signal with adaptive bit allocation," Trans. Acoustic Soc. Japan, 280-2, Apr. 1980 (in Japanese).

[14] H. Suzuki, K. Momma, and Y. Yamao, "GMSK digital portable transceiver using $32 \mathrm{kps}$ ADM, " in IEEE $33 \mathrm{rd}$ Veh. Technol. Conf. Rec., May 1983, pp. 341-346.

[15] F. de Jager and C. B. Dekker, "Tamed frequency modulation, a novel 
method to achieve spectrum economy in digital transmission," IEEE Trans. Commun., vol. COM-20, pp. 534-542, May 1978.

[16] S. Ogose and K. Murota, "Differentially encoded GMSK with 2-bit differential detection," Trans. IECE Japan, vol. J64-B. no. 4, pp. 248-254, Apr. 1981.

[17] T. Masamura et al., "Differential detection of MSK with nonredundant error correction," IEEE Trans. Commun., vol. COM-27, no. 6, pp. 912-918, June 1979.

[18] K. Hirade et al., "Error-rate performance of digital FM with differential detection in land mobile radio channels," IEEE Trans. Veh. Technol., vol. VT-28, no. 3, pp. 204-212, Aug. 1979.

[19] H. Suzuki, "Optimum Gaussian filter for differential detection of MSK," IEEE Trans. Commun., vol. COM-29, pp. 916-918, June 1981

[20] S. Ogose, "Optimum Gaussian filter for MSK with 2-bit differential detection," Trans. IECE Japan, vol. E 66, no. 7, pp. 459-460, July 1983

[21] M. K. Simon and C. C. Wang, "Differential versus limiter-discriminator detection of narrowband FM," IEEE Trans. Commun., vol. COM-31, pp. 1227-1234, Nov. 1983.

[22] — , "Two-bit differential detection of MSK," submitted for presentation at GLOBECOM'84.

[23] R. F. Pawula, S. O. Rice, and J. H. Roberts, "Distribution of the phase angle between two vectors perturbed by Gaussian noise," IEEE Trans. Commun., vol. COM-30, no. 8, pp. 1828-1841, Aug. 1982.

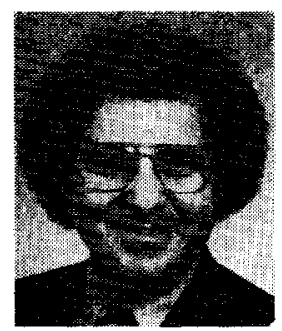

Marvin K. Simon (S'60-M'66-SM'75-F'78) received the B.S. degree in electrical engineering from the City College of New York, New York, in 1960 , the M.S. degree in electrical engineering from Princeton University, NJ, in 1961, and the Ph.D. degree from New York University, New York, in 1966.

During the years 1961 to 1963 and again from 1966 to 1968 he was employed at the Bell Telephone Laboratories, Holmdel, NJ, where he was involved in theoretical studies of digital communication systems. Concurrent with his doctoral studies between 1963 and 1966 , he was employed as a full-time instructor of electrical engineering at New York University. Since 1968, he has been with the Jet Propulsion Laboratory where he is presently a Senior Research Engineer. His research has explored all aspects of the synchronization problem associated with digital communica- tion systems, in particular, the application of phase-locked loops. More recently, his interests have turned toward the study of modulation and coding techniques for efficient spectrum utilization and spread spectrum communications.

Dr. Simon is a winner of the 1984 IEEE Centennial Medal, and a member of Eta Kappa Nu, Tau Beta Pi, and Sigma Xi. He is a winner of a NASA Exceptional Service Medal in recognition of outstanding contributions in analysis and design of space communication systems. He is also listed in American Men and Women of Science and Who's Who in Technology. In the past he has served as Editor of Communication Theory for the IEEE Transactions on Communications and Technical Program Chairman of the 1977 National Telecommunications Conference. In addition to his technical contributions in other areas, he has published over 70 journal papers on the above subjects and is co-author of the textbook Telecommunication Systems Engineering, (Englewood Cliffs, NJ: Prentice-Hall, 1973) and the IEEE Press Book Phase-Locked Loops and Their Application. His work has also appeared in the textbook Deep Space Telecommunication Systems Engineering by J. H. Yuen, Ed. (Plenum, 1983) and the IEEE Press Book Spread Spectrum Communications Ed. by C. E. Cook, F. W. Ellersick, L. B. Milstein, and D. L. Schilling. Recently he has just completed, together with J. K. Omura, R. A. Scholtz, and B. K. Levitt, the textbook Spread Spectrum Communications, to be published in 1984 by Computer Science Press, Rockville, MD. He also serves as a consultant to private industry and the government in the above mentioned areas of specialization and has participated in short courses offered by these same installations and leading universities through-out the world. In 1979-1980, he held a part-time academic position as Visiting Professor of Electrical Engineering at the California Institute of Technology.

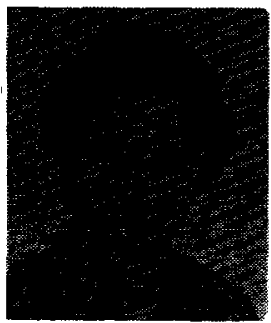

Charles C. Wang ( $S^{\prime} 80$ ) was born in Taipei, Taiwan, Republic of China, on December 14, 1952. $\mathrm{He}$ received the B.S. degree in communication engineering from National Chiao-Tung University, Taiwan, Republic of China, in 1974, and the M.S. and Engineer degrees in communication systems from the University of California, Los Angeles, in 1977 and 1979 , respectively.

From 1979 to 1981 he was with LinCom Corporation, Los Angeles, CA. Since July 1981 he has been with Jet Propulsion Laboratory, Pasadena, CA. His current research interests include coding applications, satellite communications, and spread spectrum systems. 